\title{
Study of Classical Conditioning in APLysia through The Implementation of COMPUTATIONAL MODELS OF ITS LEARNING CIRCUIT
}

\author{
A. SANtos (nino@udc.es), A. Porto (anuska@udc.es), J. Romero (jj@udc.es), A. Albó (albo@udc.es), \\ AND A.PAZOs (apazos@udc.es)
}

Faculty of Computer Sciences. A Coruña University. Spain

PHONE: +34 981167000 Ext. 1345

FAX: +34 981107160

\begin{abstract}
The learning phenomenon allows various analysis levels, but this article treats one specific paradigm of Artificial Intelligence, Artificial Neural Networks, whose main virtue is their capacity to look for unified and mutually satisfactory solutions with an important turn towards biological and psychological models. Given the fact that a substantial part of the procedures, methods, etc. proposed until the present time use the principles, models and data of biology and psychology, or both at the same time, we focus on models which look for a greater degree of coherence. That is the reason why this article analyzes and compares all the aspects comprised by two Artificial Neural Networks models whose implementation is presented: Gluck's and Thompson's, and Hawkins'. A multithread computer model is developed with the purpose of analyzing those models in order to study the simple learning phenomena in a sea invertebrate, the Aplysia Californica, and check their capacity for research in psychology and neurobiology. The predictive capacity differs significantly for both models.: the Hawkins model covers better the behavioral repertory of Aplysia, on the associative as well as the non-associative learning level. Through the integration with neurobiological and behavioral models of associative learning, the applied Artificial Neural Networks modelling technique broadens its scope, allowing the enhancement of some architectures and procedures that are being used nowadays.
\end{abstract}

Keywords: learning, classical conditioning, computational models, multithread 


\section{INTRODUCTION}

The learning phenomenon is being studied by many disciplines: the more traditional ones, such as Psychology or Biology, but also others such as the control theory, signal processing, Cybernetics or Artificial Intelligence. All these disciplines focus their attention on one basic concept: adaptation. In Psychology, the behavioral analysis is carried out largely independently from Biological analyses, which are based on a structural and functional approach that, in most cases, is limited to a local and specific reach, not allowing a global vision. The pressure of technological efficiency weighs on the computational sciences, together with an excessive reliance upon formal analyses, which makes them forget that the best systems for information processing and gathering, and for the storage and manipulation of knowledge are highly redundant, guided by practical facts, heuristic, and based on the optimisation of their behavior.

In this context, David Marr (1982) defines a general framework that is still in use today and assigns a role in the learning analysis to each of the different approaches. This model specifies a descriptive level for each discipline, together with the type of restrictions that they should mutually exercise. Consequently with this statement, the simple learning model proposed by Hawkins and Kandel in 1984, $\mathrm{HK}_{\mathrm{m}}$, and developed from a large number of works on the marine invertebrate Aplysia Californica, is an example of how the neurobiological analysis can integrate with the psychological analysis in order to generate a set of hypotheses (i.e., a circuit) that is much more complete than what each of these disciplines could generate independently. Their experimental verification will have further reaching consequences than an independent approach, which makes them much more valuable. It is precisely in this verification field that a general approach joins the computation sciences, which are able to provide the adequate methodology.

At this point, we should consider Bailey's proposal (1997) concerning the various study levels of a real system approached by several disciplines and modelled with neurons. He states that by using a vertical method we can build a bridge between the levels so that the highest levels reduce or abstract themselves to the lowest ones. It is at this point of union between disciplines that we consider, as one of the main purposes of this work, the development of an ideal environment that integrates the computational level into the study and the verification of the hypotheses of the aforementioned psycho-biological models. However, the proposed integration level should not be seen as a simple instrumental function, but rather as an important source of new architectures to 
resolve complex problems that require a certain level of learning (i.e., models that implement learning mechanisms in real time, without evaluative feedback by the environment).

The environment developed is applied as an experimental basis to two theoretical models of Artificial Neural Networks (ANNs) in view of the verification of a whole set of learning hypotheses: the Gluck and Thompson model (1987) - henceforth GTm; and the Hawkins model (1989a,b) - henceforth Hm. These models accept that they are restricted, albeit at a different level, by principles and models of both the learning psychology (in the role of the computational theory) and Neurobiology (in the role of the physical implementation level). Both models focus on an elementary associative learning manner, known as Classical Conditioning, as it is present in the marine invertebrate Aplysia Californica. We have defined a whole set of experiments that use the developed computational models as a working tool and allow us to analyze the level of adjustment of the theoretical models GTm and Hm to the learning capacities of the invertebrate Aplysia Californica. This animal combines two important circumstances:

- It is complex enough to show the most important characteristics of the classical conditioning, which means that the different theories on the nature of this learning process are relevant (e.g., they can play the role of computational theory).

- At the same time, and thanks to its simplicity, the neurobiological analysis allows us to identify consistently, in one or several individuals, the intracellular mechanisms and the structural elements of their nervous system (e.g., the implementation level can be described adequately).

The main purpose of the experimentation phase of the developed computational model is to check whether the outputs, obtained through the simulations with the implemented models, correspond with the behavior observed in Aplysia Californica and relative to the elementary associative learning phenomenon. As a working hypothesis, we will also determine to what level this correspondence takes place and which are the concrete phenomena of the Classical Conditioning that each model manages to simulate.

Gluck and Thompson propose two approaches of the model, which we have included in our implementation: RedBásica and RedTipoI. The first implemented circuit, BasicNetwork, does not include a facilitator interneuron, which makes it a very interesting way to show which aspects are implemented by this cell. The 
second circuit, NetworkTypeI, does include a facilitator interneuron, and we will see that it is more powerful than the BasicNetwork. In both cases, we will prove the capacity of the computational model to generate four fundamental phenomena of the classical conditioning: the effect of the interval between stimuli, conditioning of the second order, differential conditioning, and blocking.

The Hawkins model approaches both the non-associative and associative learning in Aplysia Californica. Our implementation of the Hawkins model allows us to analyze some of its most relevant control procedures. Nonassociative learning reflects the results that derive from the effect of the intensity of the stimulus and the effect of the presentation frequency of the stimulus and dishabituation. Within associative learning, we expose how our computational model predicts the acquisition and extinction of the excitatory conditioning; we analyze various conditions of experimental control; we study the effect of the intensity of the stimuli and the interval between tests; we observe the temporal relations between conditioned stimulus and non-conditioned stimulus (henceforth CS and NS); we show how the model predicts the conditioning of the second order; we predict shadowing and blocking, and the relationship between the CS-NS contingency and the conditioning; and finally, we present the effect of the pre-exposure of CS and NS.

\section{Behavioral and NeUrobiological Models}

A learning theory must define in detail which are the aspects of the environment that are actually provoking it, what is being learned and how this affects future behavior. Likewise, the neurobiological analysis must describe in a concrete manner all the mechanisms and processes that facilitate and support what is being observed on a behavioral level.

Thanks to the structural simplicity of these organisms and to their sufficiently rich repertory, the analysis of the learning processes in invertebrates allows us to identify easily the neural sets that control a given behavior sequence (e.g. Emptage, N.J. et al., 1996).

Hebb's postulate (Hebb, D. O. 1949), which is coherent with Pavlov's model (Miller, R.R., Matzel, L.D. 1989), turns out to be the ideal complement of the behavioral learning theory, since it functions as an integrating 
element between Neurobiology and the Adaptive Systems theory. Taking this postulate as a starting point, the various ANN models have evolved by providing the integration of some of the principles of the behavioral theory of simple learning and by assuming, to a certain degree, the restrictions of the neurobiological analysis.

This evolution takes us to the pre-activation models (priming models), which are extensions of the model of Rescorla and Wagner (1972), initially proposed by Wagner (1976, 1979; Pfautz, Wagner, 1976), and in general proposed to transform the model of Rescorla and Wagner (1972) into a real-time learning mechanism that can predict, for instance, the effects of the interval between stimuli (IBS) or the duration of the stimuli. Moreover, researchers have tried to increase the predictive power of non-associative phenomena (e.g., habituation) as well as associative phenomena (e.g., second order conditioning, latent inhibition (Wright, W., Carew, T., 1995), etc.).

From here onwards, this study will focus on the two pre-activation models, which are strongly linked to the models and theories of animal learning and which simultaneously try to adjust to the neurobiological data and models, more concretely the Hawkins and Kandel model (1984). However, the models do not reach the same consistency between levels, because they assume a different number of neurobiological restrictions:

- Although they model the structural properties of the circuits identified in Aplysia, Gluck and Thompson do not descend to the level of the biophysical properties of the neurons (e.g., ionic currents, storage and liberation of neurotransmitters, etc.). They remain on what they label as the level of cellular description: elementary algorithms that try to reproduce certain aspects of the behavior of neurons without modelling the mechanisms that generate this behavior. In this approach, the algorithmic level is closer to the computational level than to the implementation one.

- Hawkins follows a circuit which is similar to the one proposed by Hawkins and Kandel (1984): he tries to model, albeit in a very simple manner, some of the intracellular mechanisms that determine the behavior of the cell. In this case, the algorithmic level is closer to the implementation level than to the computational one. Surprisingly, the Hm solution turns out to be more consistent with this level than the GTm solution, and the result is more general than in the previous case, since it predicts a wider range of phenomena, both associative and non-associative. 
The analysis of these restrictive models focuses on some interesting differences between them. Both models are based on the Aplysia circuit introduced by Hawkins and Kandel (1984), but the Hawkins model presents two interesting differences with regard to the Gluck and Thompson model (1987) (See figures 1 and 2).

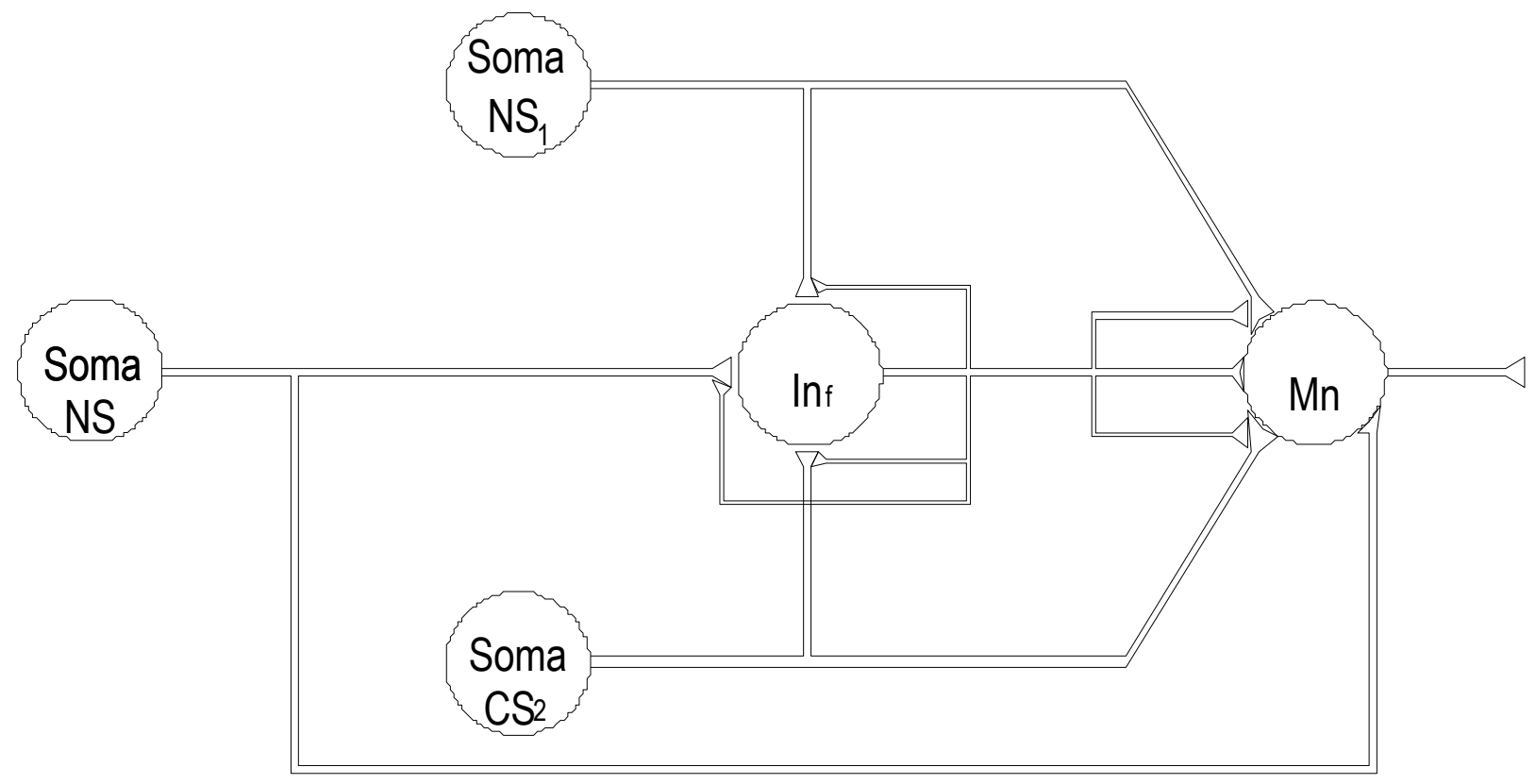

Figure 1. Circuit on which Gluck \& Thompson (1987) define a real-time learning model, compatible with the preactivation models for non-associative and simple associative learning.

- Whereas for Hawkins, all the sensory pathways are equivalent, both functionally and structurally, Gluck and Thompson define a special sensory pathway for the non-conditional stimulus (NS): It has a fixed connection (i.e., it can not be conditioned) with the motoneuron so as to maintain the efficiency of the NS, even when the interneuron has entered a refractory stage;

- For Hawkins, the facilitator interneuron does not stimulate the motoneuron directly. Gluck and Thompson, while preserving the criteria of Hawkins and Kandel (1984), implement a motoneuron double stimulation pathway: a monosynaptic pathway (where stimulus-response representations are stored (S-R)), and a polysynaptic pathway, through the facilitator interneuron (which maintains StimulusStimulus representations (S-S)). 


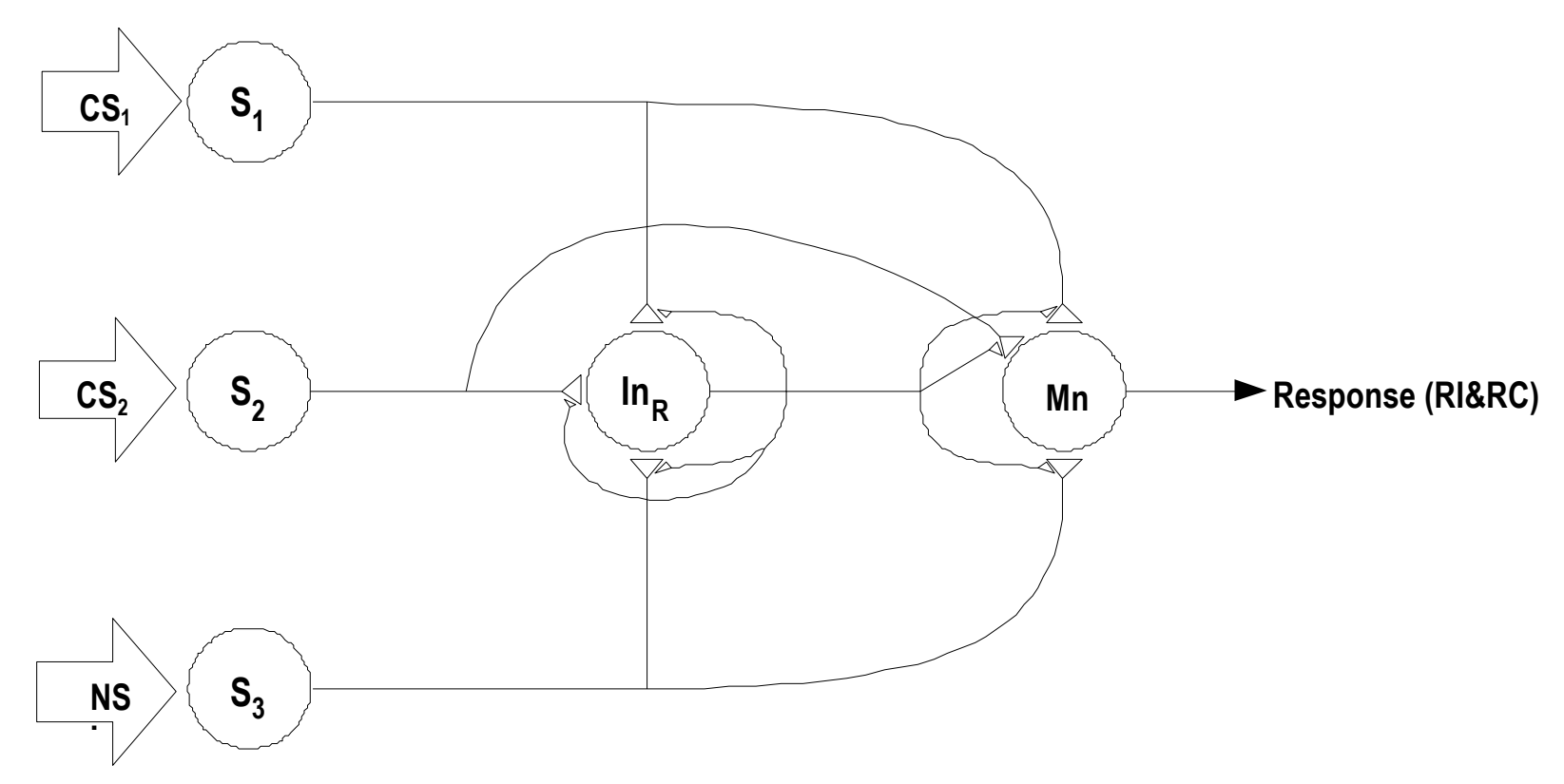

Figure 2. Circuit with which Hawkins (1989a, b) implements a preactivation model for associative and nonassociative learning.

Although the GTm adapts the Hawkins and Kandel circuit as a general framework, it remains considerably removed from the neurobiological facts it includes. Hawkins (1989a, b), on the other hand, assumes both the structure and the kind of elements that constitute it: the simple associative elements defined in models such as those of Baxter et al. (1991) and Byrne et al. (1989). The cellular processes that are implied in the conditioning studied have been simplified as follows by the Hawkins model.:

1. The majority of these processes are considered linear;

2. The habituation is only due to the inactivation of the $\mathrm{Ca}^{++}$channels, although it is proven that there are other intervening factors (Stopfer et all., 1996). Likewise, it is assumed that the sensitization is exclusively due to the widening of the action potentials.

\section{Computational MODELLing}

The most important characteristics of the developed system are the following:

- In both cases, the applied modelling tool is an object-oriented scheme (Rumbaugh, J., 1991), at the design level as well as the implementation one. 
- A multithread environment was created in the general framework provided by this object orientation. Each dynamic element of an ANN (i.e., cellular somas and synapses) is represented by an object with attributes and methods, associated to a function that allows us to manage and control its synchronization with the other elements that shape the network. This function is launched like an independent thread when the network is created in the memory. The cellular somas generate presynaptic potentials with which they activate their synapses. Apart from sending the corresponding activation signal, they will have to liberate the thread that manages them, after which the thread associated to the soma enters a waiting phase. Before it leaves this state, and given that in a soma various synapses may also converge, apart from receiving their postsynaptic potentials, the managing thread will have to wait until various events are signalled, one for each synapse it establishes.

- One of these ANN integrates not only somas and synapses, but also an additional object called MotorNetwork. This object manages the administration of stimuli, the gathering of data (i.e., activation levels of the elements) and the control of an experimental session. It is also managed by a thread (i.e., a function that is launched as an independent thread) which synchronizes it with the other elements. Figure 3 shows the general functioning of the system with the interaction between objects.

- Another object called ControlNetwork is responsible for creating the network and controlling the synchronism with the global system. Besides creating, connecting, activating, stopping, reinitiating and terminating a network, it provides:

- A list of data called TestList that functions as a source of stimuli gathered in temporal units called Tests - An area to collect the results of each simulation cycle called Gateway.

- Just like a behavioral experiment, a simulation is based on a series of tests. Each test consists of a certain number of temporal cycles. Although each cycle is considered an indivisible point, the internal structure of a network decomposes it into a series of steps (e.g., activation of sensory somas, activation of sensory synapses, activation of the facilitator interneuron, generation of sensitization signals by the facilitator interneuron, and finally, activation of the motoneuron). MotorNetwork determines the beginning and the end of a simulation cycle. Three types of stimuli can appear during a test: one NS and two CSs (i.e., $\mathrm{CS}_{1}$ and $\mathrm{CS}_{2}$ ), distinguished by the sensory soma they affect. Each test determines the start and finish times of each stimulus in terms of cycles (a stimulus that does not appear, it starts and finishes in the zero cycle). 


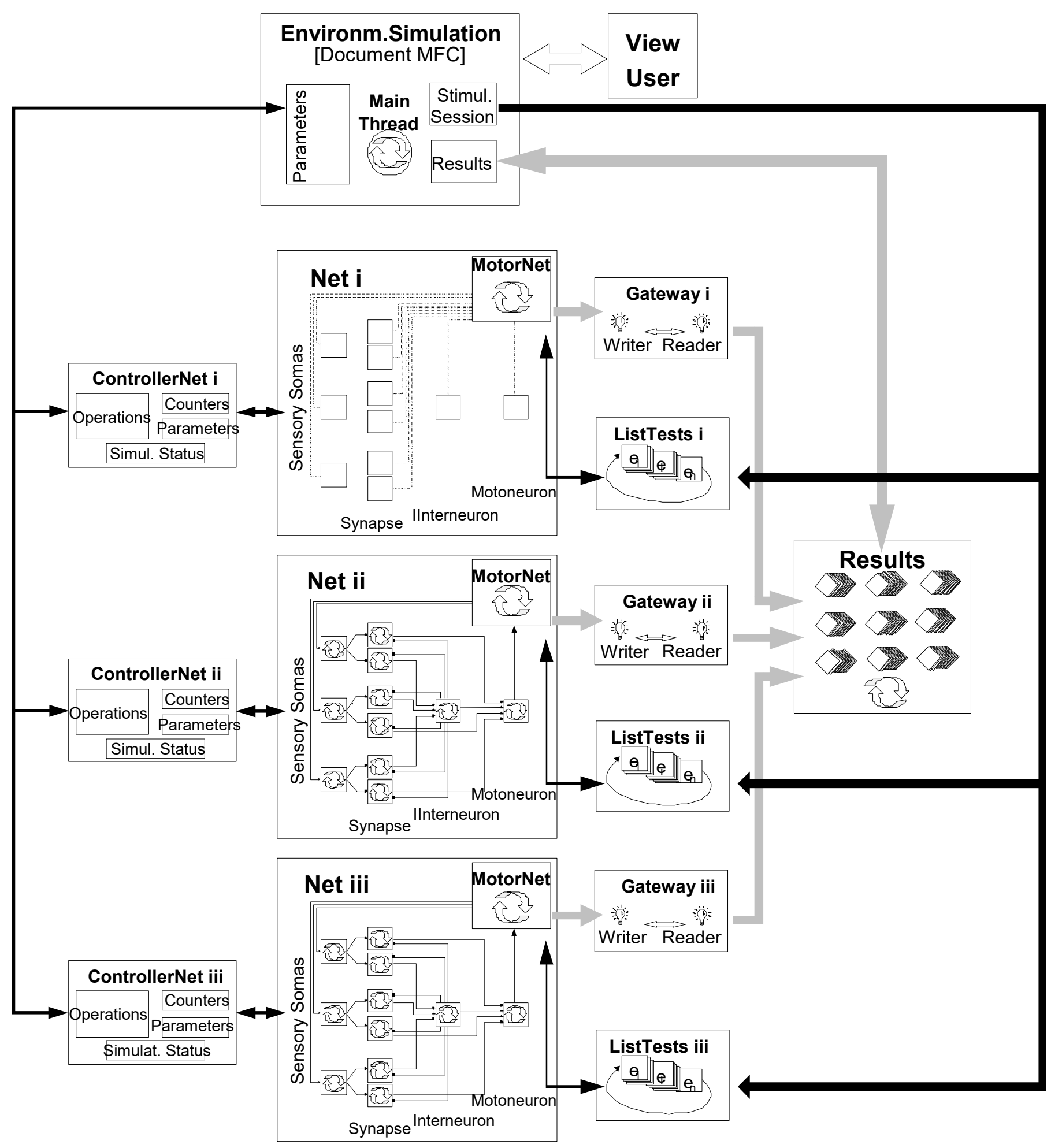

Figure 3. General scheme for the implementation of the Gluck \& Thompson models (1987) and Hawkins model (1989). A network consists of the following: stimulation connections, through which the dynamic elements (i.e., those that have an associated managing thread, symbolized by $\left(t^{-}\right)$pass on their activation values and liberate the threads of the proceses they stimulate (e.g., Network ii is in this simulation phase); and control connections, through which the object MotorNetwork updates parameters and reads values in the dynamic elements of the network (e.g., Network $i$ is in this phase). 


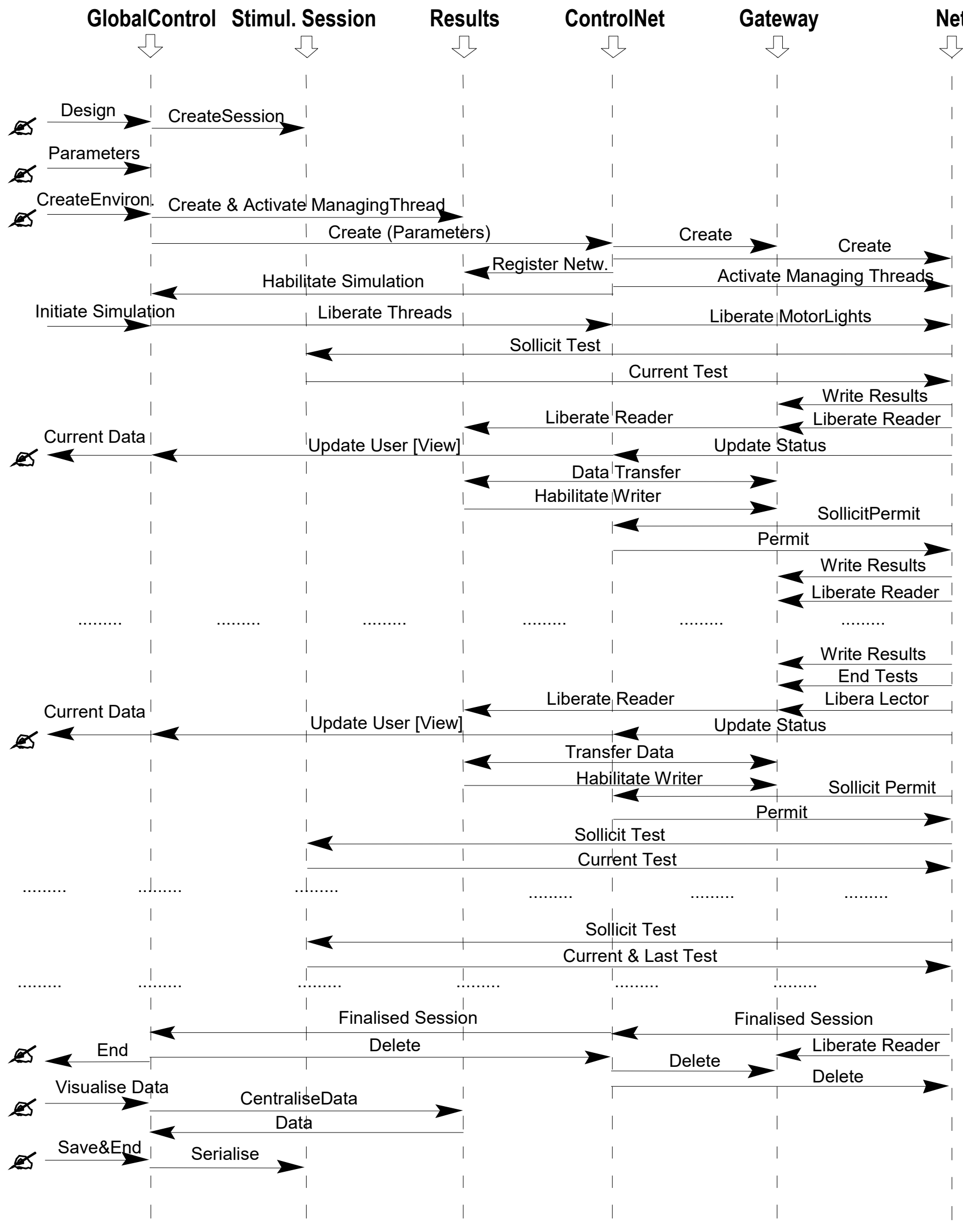

Figure 4. Scheme of events related to the general scheme of figure 3. 
- The results are stored by the network in the Gateway object, and read by an object that is managed by a synchronized thread called Results. This thread has the role of reader, whereas the previously described MotorNetwork object has the role of writer. The object Results stores multiple data of one simulation and makes them available once this simulation has ended.

- Given that most conditioning procedures imply the use of various subject groups in one single design (e.g., one experimental group and one or two control groups), a Document can simultaneously launch up to three networks, providing them with a different stimuli list. All the active networks are synchronized by the object Results. In its function of reader, it will wait until each of the Gateways has authorized the reading, which will only occur after each MotorNetwork has written the results that were generated in a simulation cycle.

The scheme of figure 4 represents a Diagram of the Layout of Events. This diagram indicates the interaction of the computational model's objects by means of a series of events. An event takes place during a certain lapse of time and transmits one-way information between two objects. The diagram shows each object as a vertical line, and each event as a horizontal arrow that goes from the transmitting object to the receiving object.

The following paragraphs will cover separately the implementation of each of the models (even though they share many aspects).

\subsection{COMPUTATIONAL IMPLEMENTATION OF THE GLUCK AND THOMPSON MODEL}

The GT model includes two connection types:

- Conditionable Synapses: they connect the sensory somas, represented in the implementation by the stimulative object, with both the facilitator interneuron and the motoneuron. They are the points where the effects of the conditioning are consolidated through a unique mechanism called presynaptic facilitation.

- Non-conditionable Synapses: unlike the previous synapses, they are not modified by experience. The model contains two non-conditionable synapses, both connected to the motoneuron: One from the sensorypathway of the NS, the other from the facilitator interneuron. In the implementation developed, the first of these fixed synapses is represented by an object that was specifically created to this effect, whereas 
the second is implemented by means of a method in the object that represents the motoneuron (i.e., it does not have an associated object).

Thanks to this configuration, two pathways are associated to each stimulus through the facilitator interneuron: a monosynaptic pathway and a polysynaptic pathway. We also create a special pathway for the non-conditioned stimulation by using a synapse whose efficiency is initially high and which is not modified in the course of the training (i.e., it connects directly the pathway of the NS with the motoneuron).

The interneuron, which represents the state of the system, plays a double role in this model. On the one hand, it is the sensitization source for all the conditionable synapses. On the other hand, by stimulating the motoneuron directly and non-conditionally (i.e., with a fixed synapse, although the activation of this cell depends on the conditioning level of the synapses that contact it), it allows associations of the S-S type.

Gluck and Thompson (1987) propose two circuits: a basic circuit, in which there is no intervention by an interneuron (i.e., its purpose is precisely to illustrate the fundamental role of this cell), and a complete circuit, which is an extension of the previous one (i.e., it includes an interneuron). Both circuits were implemented, and a number of problems appeared in both circuits with the sensitization process in the non-conditioned pathway. We shall explain these problems in detail.

In the case of the first circuit called BasicNetwork where there is no interneuron, the non-conditioned stimulation pathway is also the presynaptic facilitation source. Even though the implementation by Gluck and Thompson (1987) shows an extreme sensitiveness to the interval between stimuli (i.e., one of the soundest results offered by the experimental analysis of animal behavior), it is not something that can explicitly be derived form the formalization of the model. An implementation that exclusively follows this formalization (as seen in the first version of the implementation presented here) cannot predict, for instance, the absence of conditioning when a simultaneous procedure is used (i.e., CS and NS start at the same time). 
It is clear that a NS that lasts long enough can, from the simultaneous presentation of a CS, sensitize a synapse that enters its eligibility period. Although they do not refer to this restriction, Gluck and Thompson (1987) assume that the stimuli that are being used have a duration of a temporal cycle (at least the NS).

The developed computational model allows stimuli of a longer duration (i.e., various cycles), although functionally speaking they will behave as stimuli of one single cycle:

- The eligibility period of a synapse opens at the start of the CS and does not change according to its duration;

- The sensitization capacity of the NS is only restricted to its first cycle (i.e., this model does not predict effects of the duration of the NS, or differences between a delay procedure and a trace procedure that use the same IBS).

A different problem arose in the case of the second circuit, called NetworkTypeI. The main sensitization source is provided by the sensorypathway of the NS through the stimulation of the facilitator interneuron. The model predicts that the pathways that correspond to the CSs will gain in efficiency so as to stimulate the interneuron and function as an alternative sensitization source - causing phenomena such as conditioning of the second order. In a first version of our computational model, we followed the GTm and used a conditionable synapse to connect the NS with the interneuron (which establishes an axo-axonic contact with this synapse to sensitize it). The relevant issue is that this synapse adapts rapidly, which causes the level of synapse efficiency of the entire circuit to decrease to zero. This effect increases with the duration of the NS. This problem is due to a fundamental deficiency of the GTm: it does not implement the non-specific sensitization process, it only implements the sensitization that depends on the CS-NS match (i.e., what Hawkins and Kandel, 1984, called the amplification of the sensitization depending on the stimulation match, based on the pre-activation of a calmodulin that takes the synapse to a period of eligibility). Due to this deficiency, the implemented habituation of the NS-Interneuron synapse cannot be countered. Each time the NS presents itself, this synapse enters an eligible period, but there is no opportunity for sensitization since the NS is not followed by a stimulus, except in the reverse conditioning. This means that the synaptic efficiency of all the conditionable synapses declines as the main sensitization source declines. Efficiency will not decline in those conditionable synapses that have initiated their eligibility period before the NS-Interneuron synapse loses its efficiency. This occurs during the first tests. 
Given the fact that this model assumes a constant contraposition between gain (i.e., sensitization) and loss (i.e., habituation) of synaptic efficiency, loss will be the general trend. This loss is parallel to the NS's loss of synaptic efficiency. It is important to remember that this increase in the NS's efficiency to promote conditioning has nothing to do with the reduction models of the NS of Rescorla and Wagner (1972), with the model of Hawkins and Kandel (1984), or the models of Gluck and Thompson (1987). Paradoxically, the NS itself could counter the habituation produced by its presentation, considering the way the interneuron is connected. If the NS lasts long enough to coexist with the maximum value in the eligibility function, the activation it provokes in the interneuron will unchain sensitization signals that strengthen the synapse established by thepathway of the NS with this cell. The implementation of an interneuron that enters a refractory state when the activation goes beyond a certain threshold, prevents this from happening.

The implementation we propose in this case replaces the conditionable synapse NS-Interneuron by a nonconditionable synapse (i.e., it does not lose efficiency in the course of the conditioning tests). This increases the differences between the sensorypathway of the NS and the pathways assigned to the CSs. We could easily implement a non-specific sensitization process, but this would move our development away from the model we wish to represent, since the modification does not seem to be, a priori, relevant: the simulations of Gluck and Thompson (1987) use minimal stimuli (i.e., with a duration of one cycle), which completely reduces the habituation possibility of the NS-Interneuron synapse. From a functional point of view, the synapse would behave as a fixed synapse if the parameter that controls the habituation rate were small enough.

Our computational model has two more important aspects:

- The GT model is probabilistic. For instance, the efficiency of a synapse is defined as the probability to pass a presynaptic action potential on to the postsynaptic element. This introduces a substantial level of variability into the model. In order to represent in our system the most consistent effects of the model, we implement the possibility to realize multiple conditioning sessions with the same stimulation set. The final result will be the average of these sessions.

- The postsynaptic potentials are represented as synchronization events that pass on to the signalled state. When they are read by the postsynaptic element (which means that they wait in the synchronization object during a time zero), they automatically pass on to the not signalled state. 


\subsection{COMPUTATIONAL IMPLEMENTATION OF THE HAWKINS MODEL}

The Hawkins model (1989a, b) introduces two important structural differences with respect to Hawkins and Kandel (1984), and Gluck and Thompson (1987):

- The pathway of the NS is not different from any other stimulation pathway (i.e., any pathway could function as a non-conditioned pathway), because there are no fixed synapses.

- The interneuron does not stimulate the motoneuron directly.

Functionally, the most important differences that were added to the computational model are the following:

- The non-specific sensitization is implemented.

- A NS differs from potential CSs because of a parameter that is associated to the stimulation and that represents its intensity: the most intense stimuli function like more powerful sensitization sources (i.e., sources that stimulate the interneuron). The circuit represents the intensity of the stimuli through the generated amount of action potentials. The mechanism that implements the eligibility state of a conditionable synapse is much more elementary than the mechanism of the previous model (Hawkins, 1984).

\section{ANALYSIS OF RESULTS}

The analysis of the predictive capacity of the developed computational models has shown that this capacity is very different for each model. Gluck and Thompson (1987) do not reach the same predictive capacity as Rescorla and Wagner (1972). The Hawkins model (1989a, b), clearly oriented towards the neurobiological level, covers in a much more satisfactory manner the behavioral repertoire of Aplysia, both at the non-associative (i.e. habituation and sensitization) and the associative (i.e. pavlovian conditioning) learning level, not having such a strong compromise with any particular behavioral model.

\subsection{THE GLUCK AND THOMPSON MODEL}

It is important to stress the fact that the Rescorla and Wagner model (1972) -one of the fundamental determinants of the Hawkins and Kandel model (1984) - is the only one that predicts adequately the differential conditioning and blocking phenomena. The Gluck and Thompson model (1987), based on the model of 
Hawkins and Kandel, does not predict blocking adequately (i.e., it does not predict any effects of the competition between stimuli), although it does predict the interstimulus interval effect, conditioning of the second order and differential conditioning. The following paragraphs present these characteristics.

\subsubsection{BASICNETWORK}

\subsubsection{Effect of the interval between stimuli}

Figure 5 shows how sensitive the BasicNetwork circuit of Gluck and Thompson is to the interstimulus interval of a form that is coherent with the results of the behavioral analysis in Aplysia. An interval that is too long will make the beginning of the NS coincide with very low values of the elegibility function $\phi(t)$ (i.e., the synapse is already outside of the eligibility period), which means that the probability of generating presynaptic facilitation is already very low. The simultaneous conditioning is also ineffective, but in this case, the value of $\phi(t)$ is zero (i.e., the synapse has not entered the eligibility period yet). An optimal SOA (i.e., Stimulus Onset Asynchrony interval between stimuli) will make the arrival of the NS coincide with the maximum in the eligibility function $\phi(\mathrm{t})$

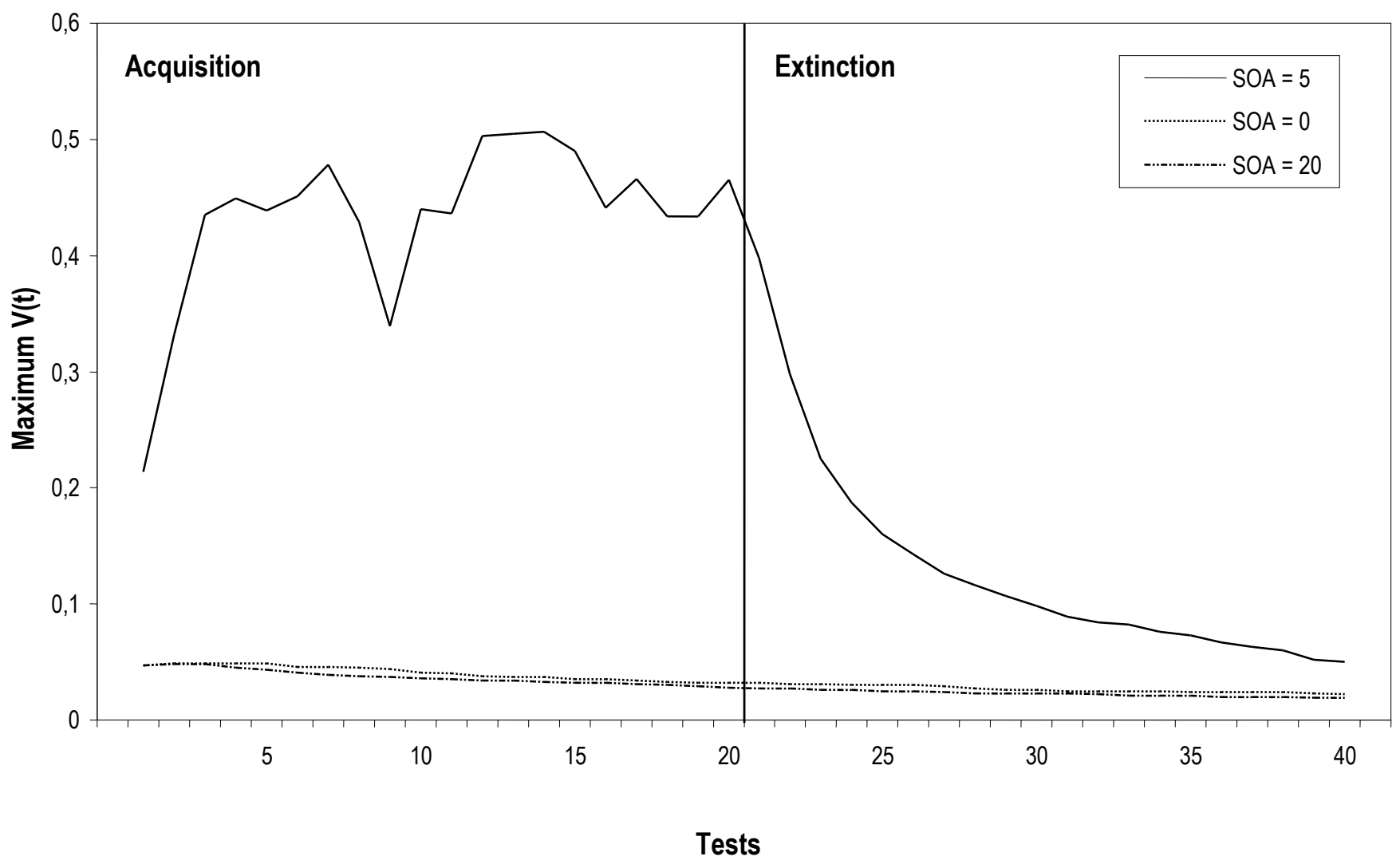

Figure 5. Effect of the interval between stimuli (SOA) in BasicNetwork. The graphic shows a procedure divided in two phases: the first phase consists of 20 reinforced tests, the second one consists of another 20 extinction tests. The simulation includes three networks with the same procedure, but with a different $\mathrm{SOA}$, as shown in the graphic. The two parameters that define the shape of 
function $\phi(t)$, have in this case the following values: $\theta=0.15, T\left(t_{E C O n}\right)=1.5$. Both the CS as the NS have a duration of 5 gycles; in all the cases, the CS starts in cycle 20, and the tests consist of 60 cycles. [Parameters: $\beta_{1}=0.4, \beta_{2}=0.2, \delta_{1}=0.85, \delta_{2}=0.6$, $\left.\mathrm{R}_{\mathrm{f}}=0.05, \mathrm{U}_{\mathrm{r}}=0.6\right]$.

\subsubsection{Differential conditioning}

The differential conditioning procedure shows the effect of the CS-NS contingency in one experimental subject.

In general, we use two stimuli: $\mathrm{CS}_{1}$ shows a positive contingency with the NS (i.e., $\mathrm{p}\left(\mathrm{EI} \mid \mathrm{EC}_{1}\right)>\mathrm{p}\left(\mathrm{EI} \mid \operatorname{notEC}_{1}\right)$ ), $\mathrm{CS}_{2}$ shows a negative contingency (i.e., $\mathrm{p}\left(\mathrm{EI} \mid \mathrm{EC}_{2}\right)<\mathrm{p}(\mathrm{EI} \mid$ notEC 2$\left.)\right)$. Figure 6 shows that BasicNetwork behaves coherently with the behavioral results (i.e., with a differential conditioning according to the CS-NS contingency).

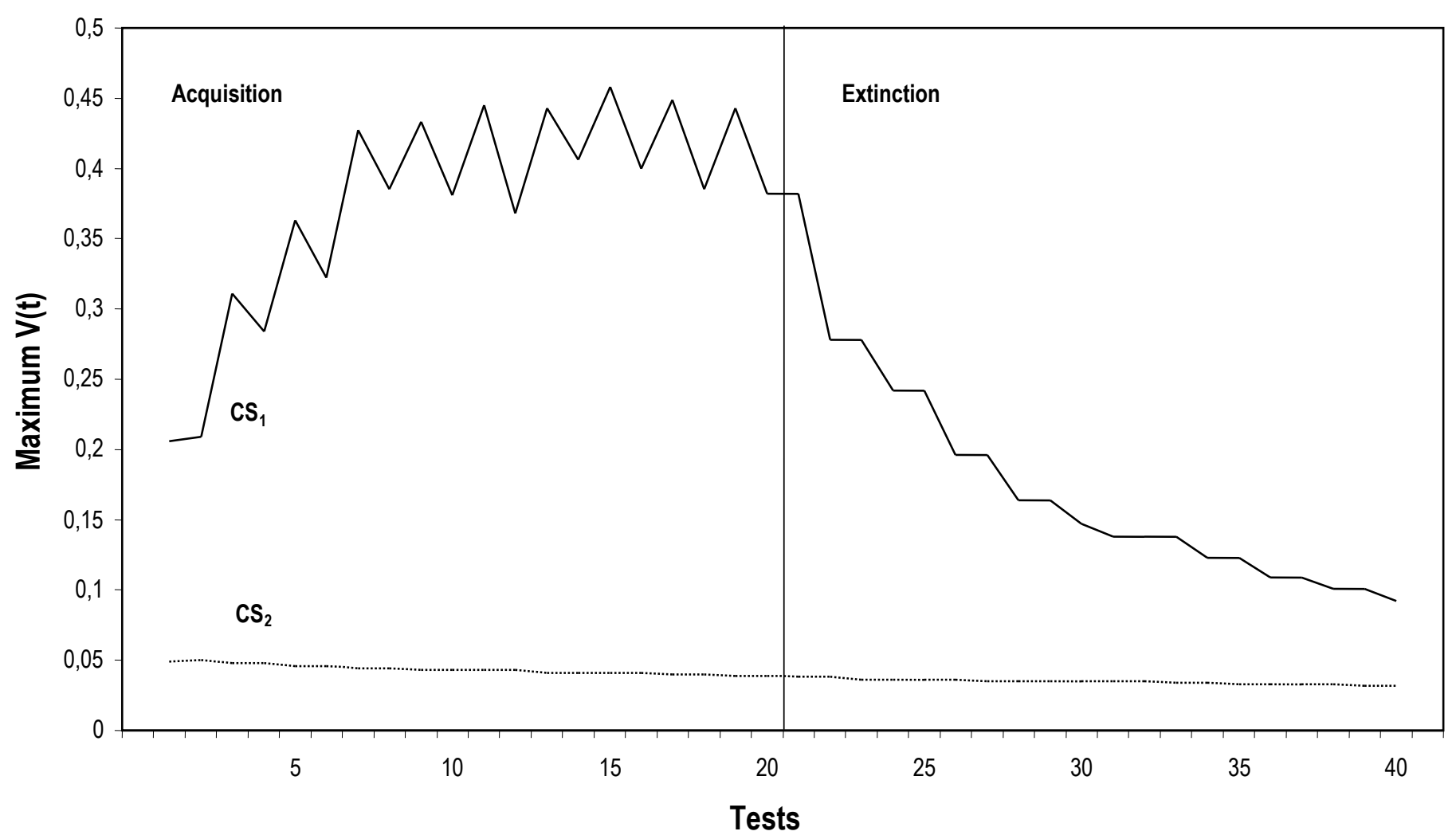

Figure 6. Differential Conditioning in BasicNetwork. This procedure implies the alternation of tests in which a $C S_{1}$ is reinforced with the presentation of a NS (test $\left.s_{1}\right)$, and non-reinforced tests that present a $C S_{2}$ alone (test $s_{2}$ ). In this case, two phases occurred. The first phase, the acquisition, consists of an $s_{1}-s_{2}-s_{1}-s_{2}-\ldots$ alternation, and it administers up to 20 tests. The second phase, the extinction, alternates non-reinforced tests, both for the $C S_{1}$ as for the $C S_{2}$. [Parameters: $\beta_{1}=0.4, \beta_{2}=0.2, \theta=0.15, \delta_{1}=0.85$, $\left.\delta_{2}=0.6, \mathrm{R}_{\mathrm{f}}=0.05, \mathrm{U}_{\mathrm{r}}=0.6\right]$.

\subsubsection{Conditioning of the second order}

The conditioning of the second order is one of the most important properties of the Pavlovian conditioning: A stimulus cannot only acquire the control of a response, it can also promote other neutral stimuli to play the role 
of conditioned controllers (i.e., a conditioned stimulus can function as a NS). The neurobiological analysis in Aplysia shows how presynaptic facilitation is the the fundamental mechanism for the classical conditioning. BasicNetwork is implemented in such a way that only the sensorypathway of the NS can exercise this kind of influence. Logically (as shown in figure 7) we should not expect a conditioning of the second order.

On the basis of this deficiency, we can estimate one of the fundamental roles of the facilitator interneuron implemented in NetworkTypeI: distribute sensitization signals (i.e. presynaptic facilitation) to and from the various sensory pathways.

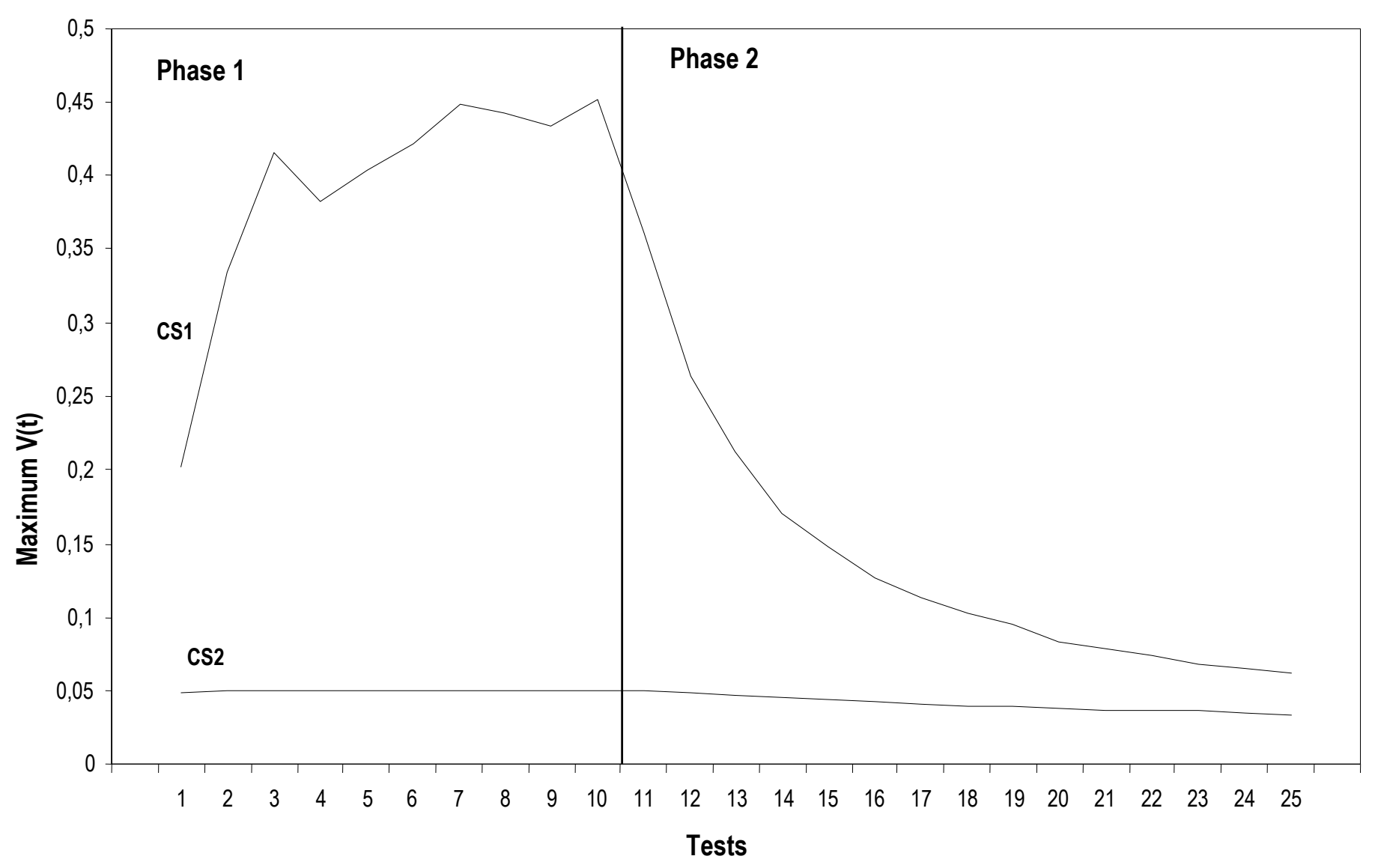

Figure 7. Absence of conditioning of the second order in BasicNetwork. The first phase of this procedure includes 10 reinforced $C S_{1}-N S$ tests. The synaptic efficiency (i.e., $\left.V(t)\right)$ of the sensory pathway of $C S_{1}$ increases in the course of the session (i.e., excitatory Pavlovian conditioning is generated). The second phase consists of another 10 tests in which the previously conditioned CS 1 plays the role of reinforcer when presented after a neutral stimulus $C S_{2}$ (i.e., tests $C S_{2}-C S_{1}$ ). Although we can observe the extinction of the $C S_{1}$, the synaptic efficiency of the sensory pathway of the $C S_{2}$ does not increase. [Parameters: $\beta_{1}=0.4, \beta_{2}=0.2, \theta=0.15$, $\left.\delta_{1}=0.85, \delta_{2}=0.6, \mathrm{R}_{\mathrm{f}}=0.05, \mathrm{U}_{\mathrm{r}}=0.6\right]$.

\subsubsection{Blocking}

As in the previous case, blocking is a fundamental phenomenon of classical conditioning. However, since BasicNetwork cannot generate blocking (cf. figure 8), the facilitator interneuron of NetworkTypeI will have to 
assume this task. Hawkins and Kandel (1984) hypothesized that this cell could determine the blocking of a sensorypathway through its capacity to enter a refractory state.

Therefore, and summarizing the two previous points, the interneuron will have to:

- Interconnect with all the sensory sources in order to distribute conveniently the sensitization signals.

- Stop these signals from going into a determined sensory pathway in the typical circumstances provoked by blocking, as we can observe at the behavioral level (i.e., when this sensory pathway provides information that is contextually redundant).

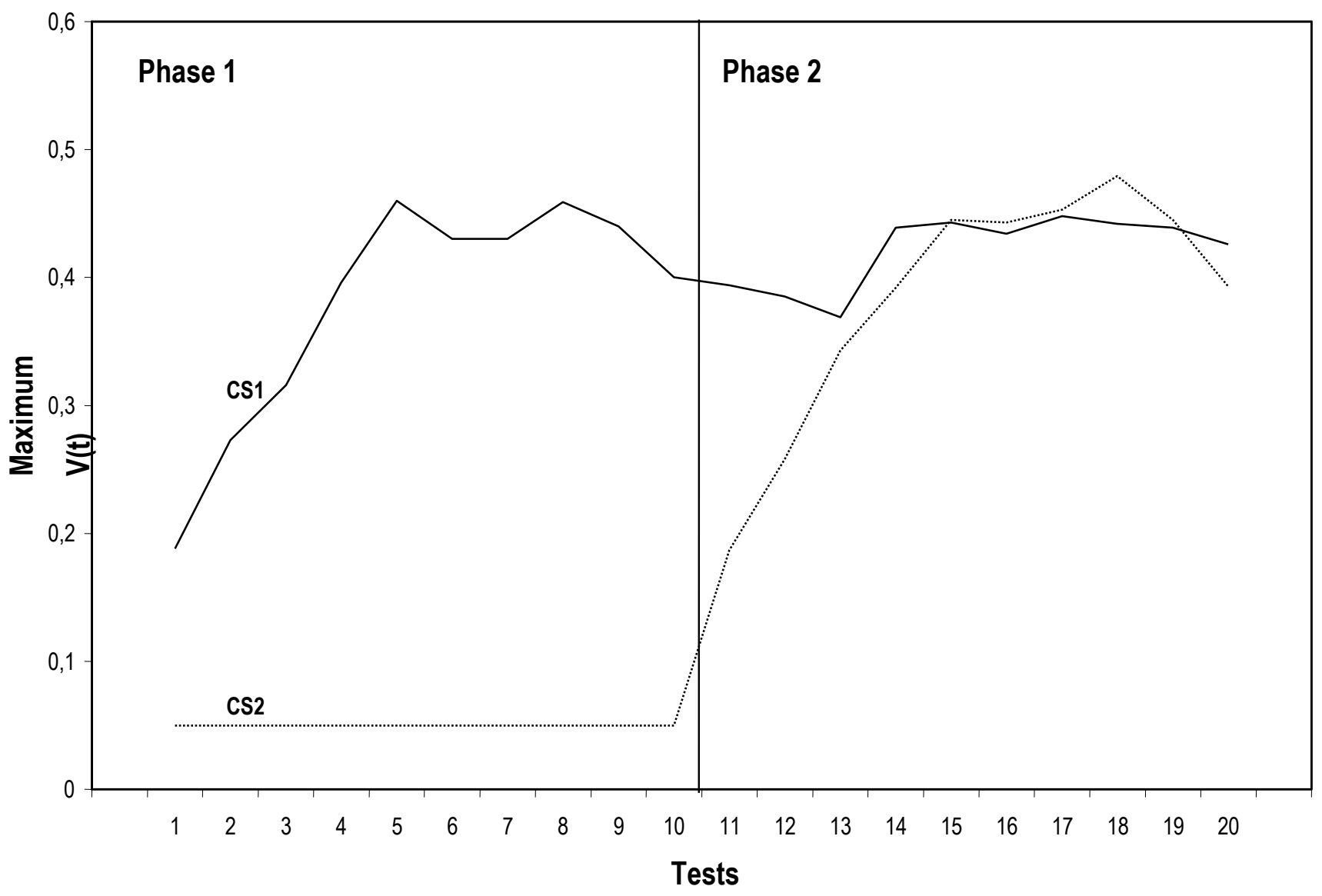

Figure 8. BasicNetwork does not predict blocking in the conditions in which Aplysia shows it. The procedure is divided into two phases: The first one consists of 10 reinforced CS $1-N S$ tests. The second phase introduces a second stimulus, CS 2 , which forms a stimulative compound with the previously conditioned $C S_{1}$ (i.e., they start and end together), during another 10 tests. Under these circumstances, Aplysia generates a lower conditioning level -blocking - for the added stimulus, CS 2 (i.e., for the stimulus that, according to Kamin and Rescorla \&o Wagner, is redundant, since its appearance does not introduce any contextual changes which were not predicted by the $C S_{1}$ ). 


\subsubsection{NETWORKTYPE I}

This network includes a facilitator interneuron that, at least a priori, covers the two functions that were specified in the analysis of BasicNetwork.

\subsubsection{Effect of the interval between stimuli and differential conditioning}

Figure 9 shows how this network generates both phenomena, just like the BasicNetwork. As was the case with the BasicNetwork -we are using the same procedure (see figure 5) -, only the SOA with 5 cycles produces a significant conditioning; however, NetworkTypeI shows in the other two procedures (SOA 0 and SOA 20) a remarkable variability in the evolution of the synaptic efficiency which contrasts with the values obtained with BasicNetwork. The values shown in figure 9 for these two cases are the result of the intervention of one single non-associative process: habituation. Both the simultaneous SOA and the one that surpasses the effective eligibility period impede presynaptic sensitization, the single mechanism that can increase the synaptic efficiency in the model of Gluck and Thompson (1987).

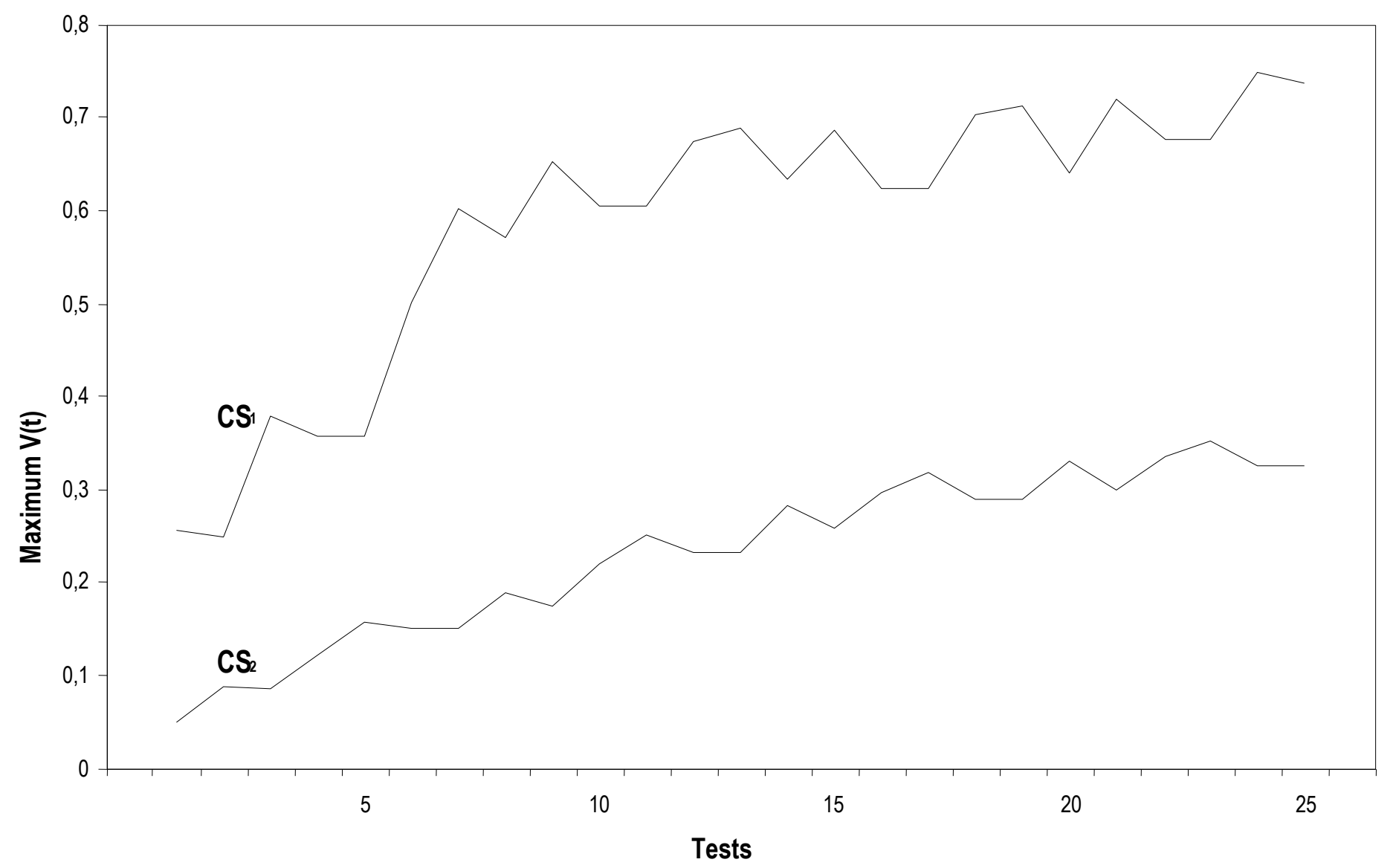




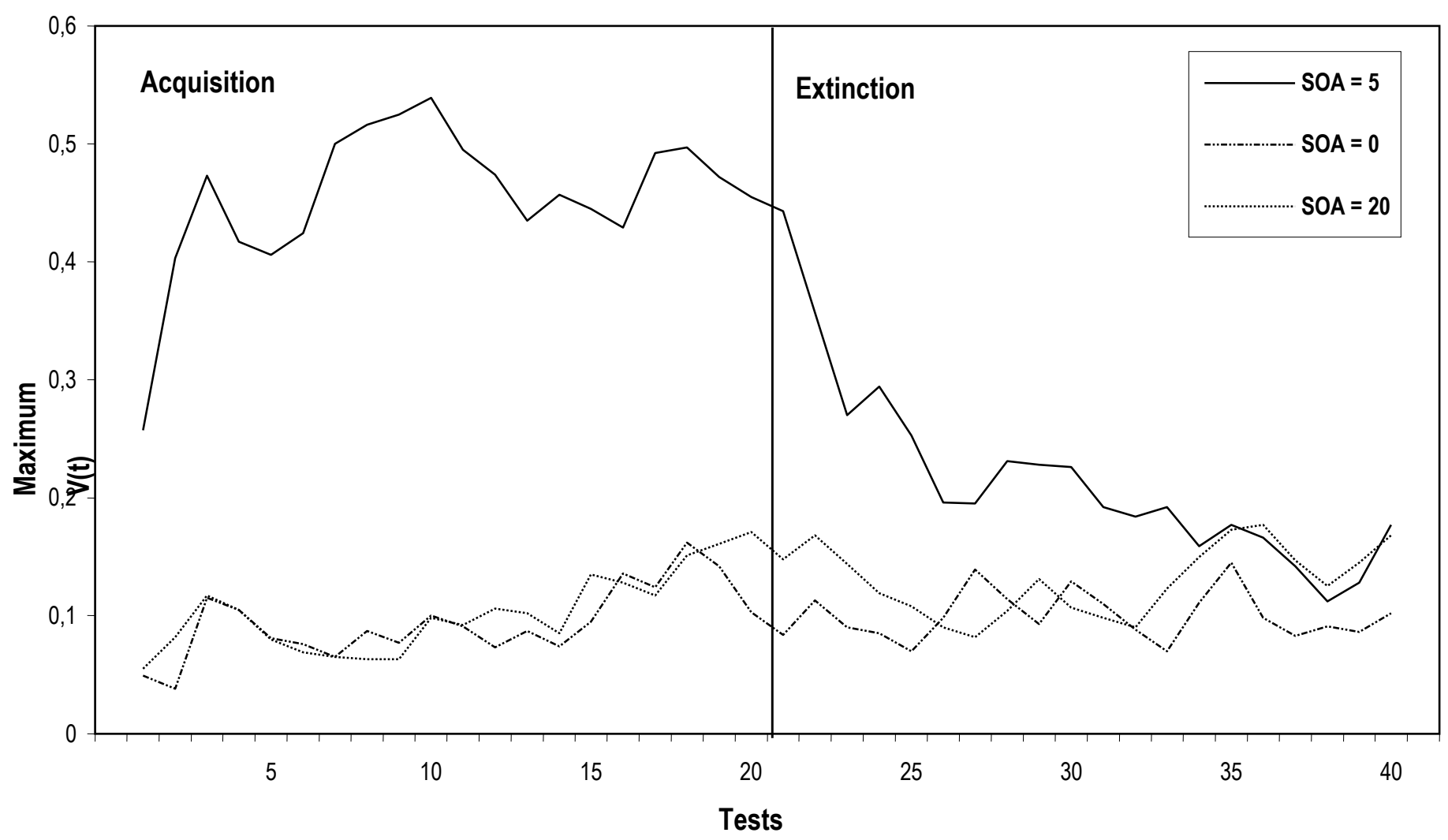

Figure 9. Effect of the interval between stimuli (upper graphic) and differential conditioning (lower graphic) shown by NetworkTypeI.

The facilitator interneuron of NetworkTypeI generates a behavior that is constantly controlled by the habituation as well as by the presynaptic sensitization. When the SOA is optimal, the sensitization exceeds the habituation systematically. With a SOA that is not optimal, this relationship ceases to exist, but the interneuron can occasionally generate sensitization signals as long as its activation is not strictly zero. The increase of the interneuron activation depends on two factors: its possible refractory state and the existence of postsynaptic excitatory potentials. However, both factors indirectly control the generation of action potentials (i.e., sensitization signals) In a given cycle, the interneuron generates action potentials based on one single criterium: the value of $\mathrm{A}_{\mathrm{If}}(\mathrm{t})$, although it has not been stimulated or it is in a refractory state; the factors increase or decrease the value of this function in proportion to its actual value, but they do not directly determine the generation of potentials. The interneuron that is in a refractory state and $\mid$ or not stimulated can sensitize an eligible synapse, although this is not likely to occur. A synapse leaves its eligibility state gradually, so that after 20 cycles it can be sensitized, but this does not usually happen. Both factors contribute to the creation of an 'activation reserve' in the system, which leads to the 'behavioral noise' of figure 9. 


\subsubsection{Conditioning of the second order}

As shown in figure 10, the facilitator interneuron added to NetworkTypeI covers one of the two functions that were previously assigned to it: the distribution of the sensitization signals to the entire system, so that other sensory pathways which are not those of the NS can promote this sensitization (i.e., generation of conditioning of a superior order).

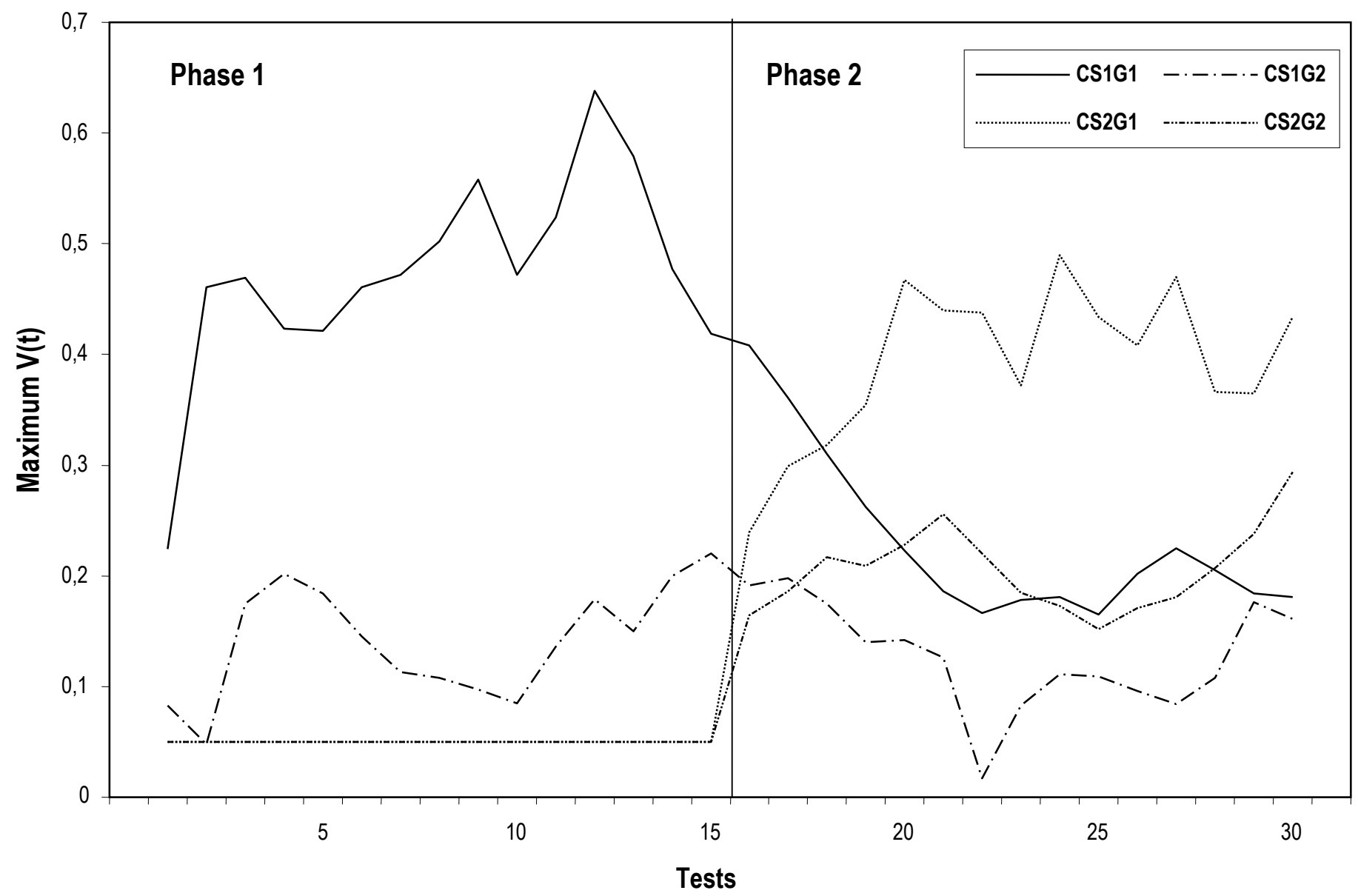

Figure 10. Conditioning of the second order in NetworkTypel. The procedure shown includes two phases administered to two different networks. The first network, which functions as an experimental group-G1-, receives during the first phase 10 optimal couplings $C S_{1}-N S$, followed by a second phase with 10 optimal couplings $C S_{2}-C S_{1}$ (i.e., the CS1 acts as a reinforcer). The second network, which functions as a control group-G2-, receives during the first phase 10 tests in which the CS 1 precedes or follows the NS with a non-optimal interval $(60 \mathrm{cycles})$ and a second phase identical to the $G 1$ one. The differences in the interval between stimuli cause the $C S_{1}$ to condition itself during the first phase in G1, but not in G2. In this way, CS 1 acquires the capacity to function as a reinforcer (i.e., conditioned reinforcer) during the second phase of the procedure, thus provoking an increase in the synaptic efficiency of the sensory pathway of the $C S_{2}$, but only in $G 1$.

\subsubsection{Blocking}

Figure 11 clearly shows how NetworkTypeI does not predict blocking. As a result, although the interneuron is able to distribute controlled excitement signals by conditioned reinforcers, it cannot suppress them in the 
circumstances under which blocking is usually observed. The original idea of Kandel and Hawkins (1984) consisted of assuming that the interneuron, due to the stimulation of the pretrained NS1, would enter a refractory state in order to avoid that the ulterior arrival of the NS would provoke the necessary sensitization signals to promote the conditioning of the sensorypathway of the CS2. However, Gluck and Thompson's version of this model does not exactly behave in this way.

First of all, Gluck and Thompson define 'refractory state' as follows: $\mathrm{R}_{\mathrm{f}}(\mathrm{t})$ is a function that takes value $1-$ it normally takes value zero- when the interneuron exceeds a certain activation threshold; from that moment onward, it decreases exponentially and returns to value zero. $\mathrm{R}_{\mathrm{f}}(\mathrm{t})$ regulates the activation of the interneuron and makes it behave as if it had not been stimulated, with a probability that equals the value that $\mathrm{R}_{\mathrm{f}}(\mathrm{t})$ takes in a determined cycle (i.e., it promotes a decrease of the activation that is proportional to its actual value: $\left.-\delta_{2} A_{\text {If }}(t)\right)$, but it does not prevent the interneuron from generating action potentials. As a result, the'refractory state' does not necessarily imply a neuronal silence. As the cycles elapse, the probability (i.e., 1- $\mathrm{R}_{\mathrm{f}}(\mathrm{t})$ ) that the interneuron increases its activation grows (as a matter of fact, after the first cycle of refractoriness, where $R_{\mathrm{f}}(t)=1$, the interneuron could increase its activation level in all the stimulation cycles, contrary to what we usually expect from refractoriness). We would expect that, in a design such as in figure 11, in which the NS starts five cycles after the interneuron enters its refractory state, and lasts another five cycles, the interneuron would not remain silent (i.e., it would generate sensitization signals that increase the efficiency of the synapse of the added stimulus). Moreover, considering the fact that we cannot restore a refractory state (i.e., $\mathrm{R}_{\mathrm{f}}(\mathrm{t})$ takes value 1 ), we cannot expect a process so restrictive as blocking to take place -at least not in the shape of the Rescorla and Wagner model (1972).

Secondly, this model is asymmetric with respect to the increases and decreases of the synaptic efficiency: Synapses with little efficiency show increases (i.e., $\Delta \mathrm{V}_{\mathrm{EC}}(\mathrm{t})=\beta_{1}\left[1-\mathrm{V}_{\mathrm{EC}}(\mathrm{t})\right]$ ), whereas synapses that are very efficient show decreases (i.e., $\Delta \mathrm{V}_{\mathrm{EC}}(\mathrm{t})=-\beta_{2} \mathrm{~V}_{\mathrm{EC}}(\mathrm{t})$ ). One of the consequences of this situation seriously affects the blocking. We assume that the efficiency of the pretrained CS1 is an indispensable condition for the generation of blocking, since it must excite the facilitator interneuron so that this can enter a refractory state. Paradoxically, as soon as the CS1 reaches this capacity, it tends to lose it. Besides, the general reduction of the sensitization levels caused by the refractoriness of the interneuron negatively affects the efficiency of the CS1 itself. At the exact moment when the decreases are at their peak, the signals that generate increases dissapear, 

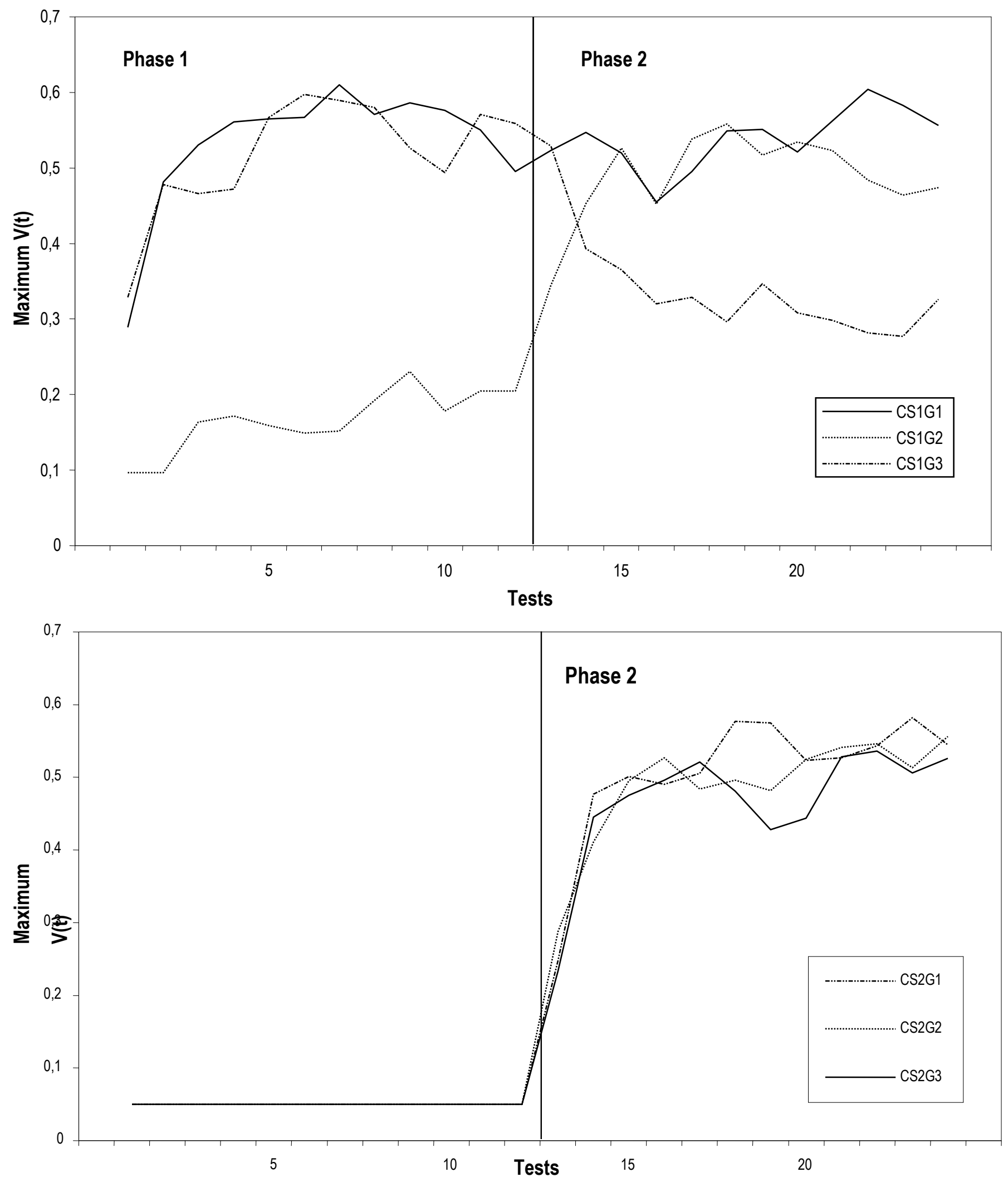

Figure 11. NetworkTypeI does not generate blocking in a design that generally does not produce it, neither in Aplysia nor in a large number of species. In this case, three networks are being used. One of them, which functions as an experimental group -G1-, receives a stimulative sequence that normally generates blocking. The other two function as control groups, since their stimulative sequences do not generate blocking. The procedure is divided into two phases of 12 tests. For G1, the first phase consists of the optimal coupling of $C S_{1}-N S$ (i.e., using an optimal SOA). During the second phase, a $C S_{2}$ is introduced which forms a stimulative compound with the pretrained CS 1 (i.e., they start and finish together), followed by the NS with the same interval as in the first phase (i.e., $\left[C S_{1}+C S_{2}\right]-N S$ ). Both control groups introduce a variant of this scheme that is supposed to affect the blocking. $\mathbf{G} 2$ is different in that, during the first phase, $C S_{1}$ and NS do not couple optimally: During half of the tests, the CS 1 precedes the NS in 20 cycles; during the other half, the NS precedes the CS 1 with the same number of cycles. $\mathbf{G} 3$ receives a first phase that is 
identical to G1, but during phase 2 the $C S_{1}$ does not form a stimulative compound with the $C S_{2}$ : While this last stimulus couples with an optimal interval with the NS, the CS, presents itself 20 cycles earlier -in the middle of the cycles- or 20 cycles later. These manipulations make us expect the absence of blocking in G2 and G3, but we should observe it in G1. However, all three groups behave in the same way: No blocking is present at all.

these increases being the elements which could counteract the habituation up to a certain extent, even though they are smaller. The result of all this is that the efficiency of the CS1 fluctuates between decreases and increases (i.e., it does not reach an asymptotic level in the sense of Rescorla and Wagner, 1972).

On the other hand, things look better for the CS2, the stimulus that was added during the second phase. The sensitization signals will not completely dissapear, and although they are scarce, the increases of synapse efficiency are substantial, which makes the interneuron condition itself. The combined action of these factors allows us to expect an absence of blocking.

What we have seen so far leads us to the conclusion that Gluck's and Thompson's (1987) approach does not seem very efficient. What they call 'description at the cellular level' can be reduced to the mere attribution of more or less elaborate algorithmic properties to the cellular elements of Hawkins' and Kandel's minimal circuit (1984), which is more coherent with a behavioral explanation level than with the neurobiological level that is generating the relevant data. The fact that certain modifications of the initial statement may correct the deficiencies of the model only accentuates the "arbitrariness" of the used approach. (When a model saves its deficiencies by growing ad hoc, it runs the risk of succeeding in results ((i.e., it behaves in the same way as that which is modelling itself)) at the expense of the real mechanisms that produce these results. This critique is often heard from empiric-experimental disciplines such as psychology, and directed to technical-formal disciplines such as the 'strong' symbolic Artificial Intelligence: Usually, the latter propose brilliant algorithms from an engineering point of view, but which are useless for the comprehension of what is supposedly being modelled).

We shall now analyze the model of Hawkins (1989a, b). The range of adequately generated phenomena suggests that this may be a more productive approach to understand the nature of the mechanisms that are responsible for the simplest learning forms. 


\subsection{THE HAWKINS MODEL}

The following paragraphs present in a separate way and by means of various graphics the results that were obtained through the implementation of the Hawkins model.

\subsubsection{NON-ASSOCLATIVE LEARNING}

The Hawkins model (1989a, b) implements the two fundamental non-associative learning forms, which are habituation and sensitization. Habituation is an intrinsic monosynaptic process (decrease of the number of active $\mathrm{Ca}^{++}$channels), whereas sensitization is an extrinsic polysynaptic process (presynaptic facilitation) (Bunge et al., 1997). Present since the Hawkins and Kandel model (1984) (this model integrates the ideas of Groves and Thompson (1970) about an E-R system and a State system, responsible for the habituation and sensitization of a response, respectively), both processes act simultaneously to generate a response level that is the algebraic sum of both. Also, while implementing a recovery mechanism of inactive $\mathrm{Ca}^{++}$channels, the model predicts phenomena that are as important as the spontaneous recovery of the habituation. Figure 12 shows an example of habituation, spontaneous recovery and rehabituation.

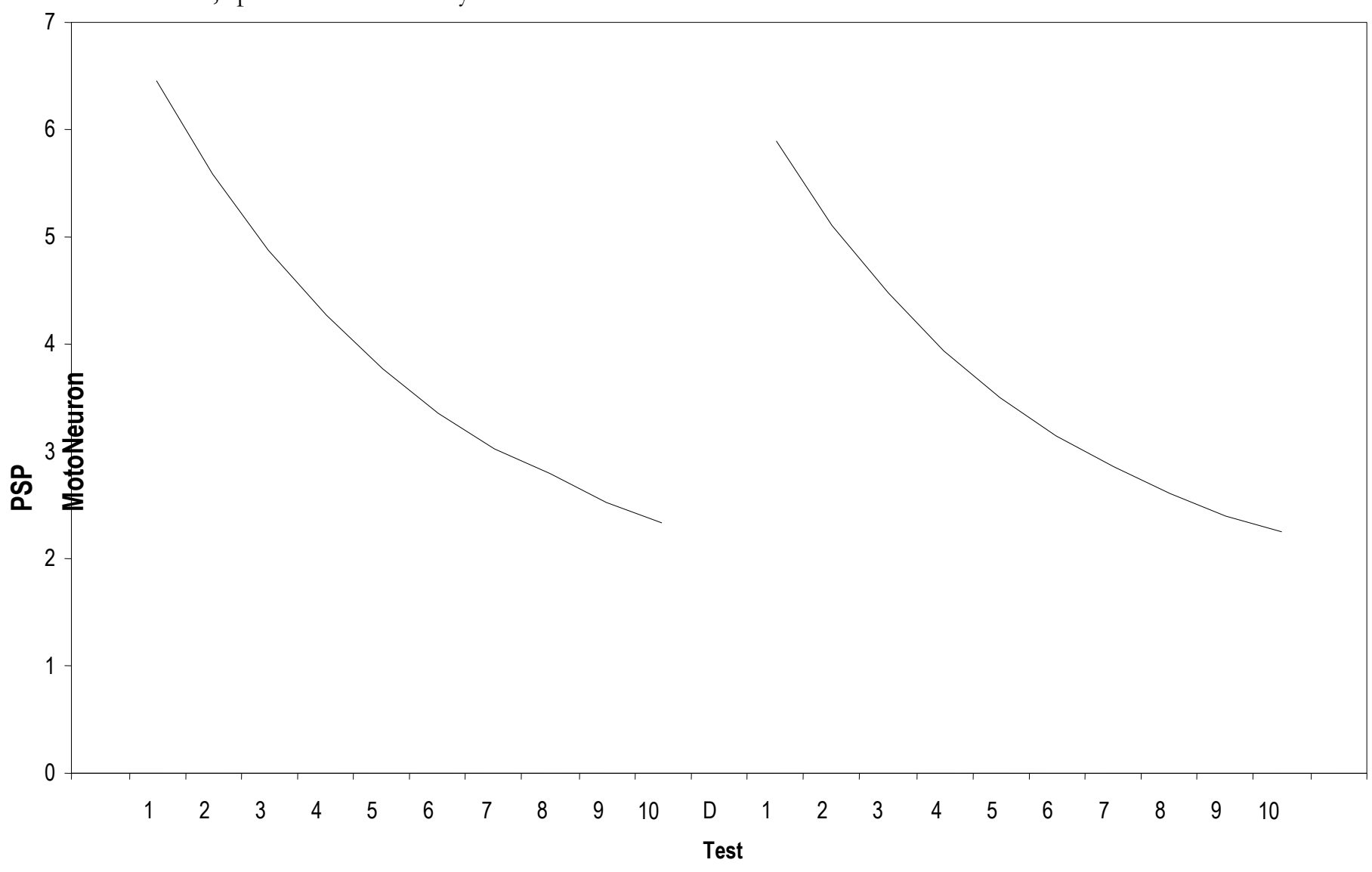

Figure 12. Habituation, spontaneous recovery and rehabituation. The procedure consists of 10 presentations of a stimulus with intensity 1 and a duration cycle (10 seconds). The series of 10 stimuli is repeated after a rest period of approximately one hour. 
Contrary to what is usually observed in animals, the model does not predict a faster rehabituation than the initial habituation. Quite on the contrary, it adequately predicts three important phenomena: The effect of the intensity of the stimulus, the effect of the stimulation frequency, and dishabituation.

\subsubsection{The effect of the intensity of the stimulus}

The habituation level is usually smaller as the intensity of the habituating stimulus increases (i.e., the balance between depression and activation of the synaptic efficiency tends towards sensitization).
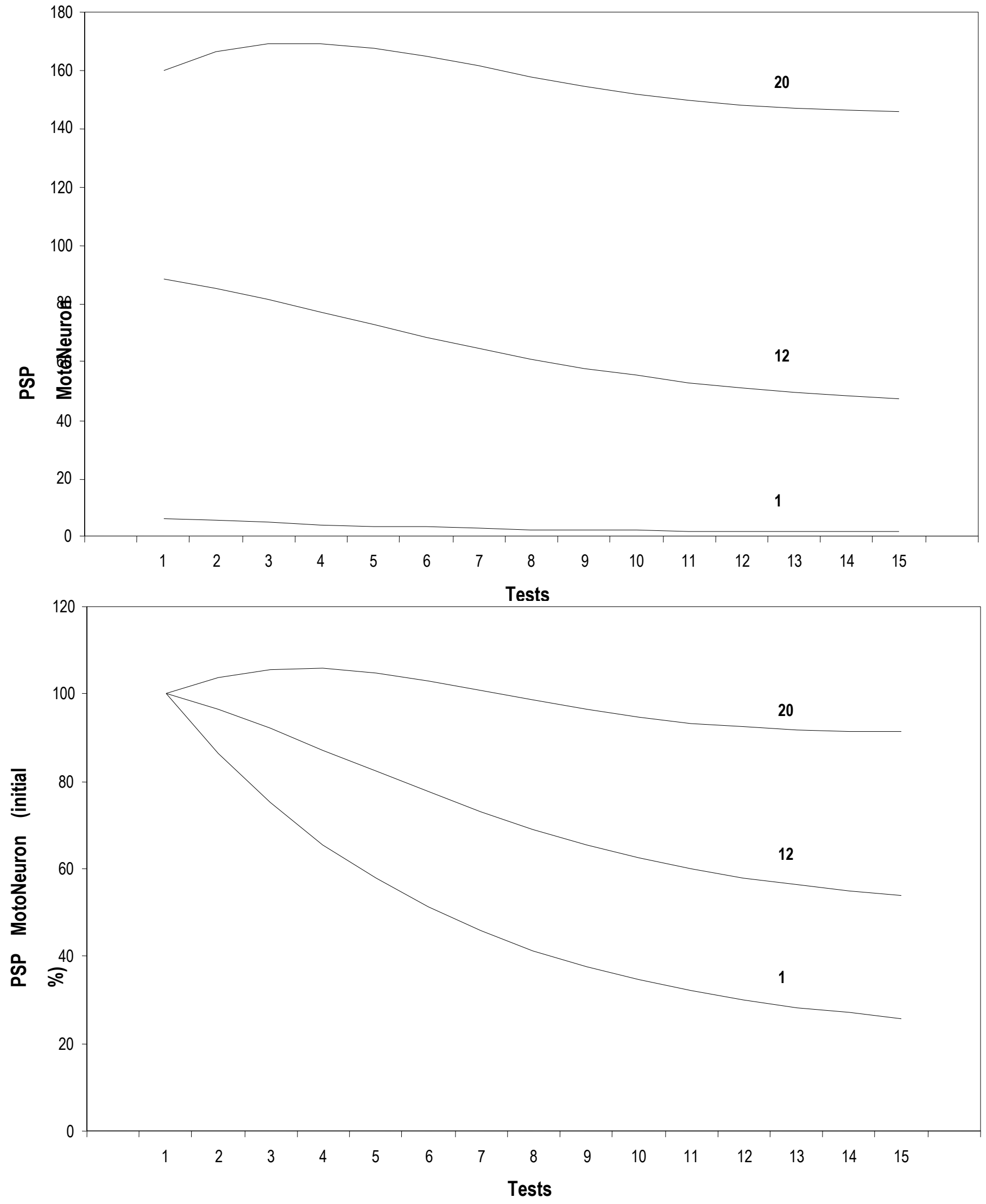


\begin{abstract}
Figure 13. Effect of the intensity of the stimulus on the babituation. The procedure uses three intensities (1, 12 and 20 arbitrary units) for a stimulus with the duration of one cycle, which is presented in 15 occasions, with an interval between stimuli of 1 minute. The upper chart shows the response of the motoneuron to each of these stimuli (PSP: postsynaptic potential). The lower graphic shows the same data, but related to the response given to the first stimulus of the series.
\end{abstract}

Figure 13 shows how the model behaves when the intensity of the habituating stimulus is altered. As the intensity of the stimulus increases, so does the force of the response of the motoneuron, whereas the habituation decreases (counteracted by the non-specific presynaptic facilitation (sensitization)). In fact, an intensity of 20 arbitrary units initially provokes a net sensitization, rather than a habituation.

\title{
4.2.1.2 Effect of the presentation frequency of the stimulus
}

The stimulation frequency can determine two very different forms of habituation: in the short term, a profound habituation that is generated by high frequency stimulation, and in the long term, a less profound habituation that is generated by low frequency stimulation and has a long duration (i.e., more resilience to spontaneous recovery). The model of Hawkins (1989), based on the recovery mechanism of inactive $\mathrm{Ca}^{++}$channels, can simulate the effect of the stimulation frequency, but it does not distinguish between habituation in the short and long terms (i.e., the recovery rate is the same, regardless of the stimulation frequency). Figure 14 reflects these results.

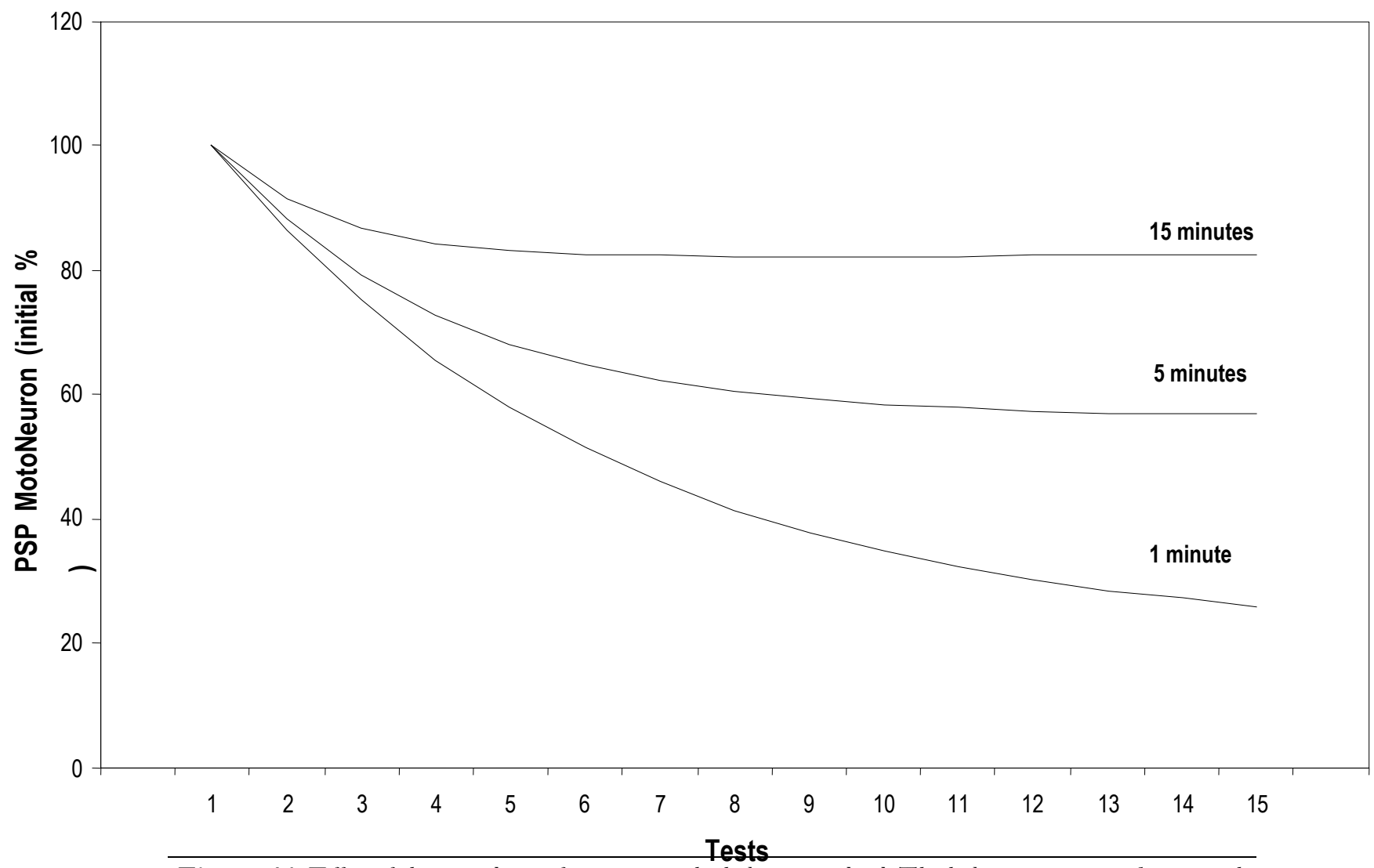

Figure 14. Effect of the stimulation frequency on the babituation level. The habituation grows deeper as the stimulation frequency increases. A stimulus with a duration and intensity yycle of 1 was used in all the cases. 


\subsubsection{Dishabituation}

A habituated response can recuperate if the animal is stimulated with an extra stimulus of a relatively big intensity. This phenomenon, known as dishabituation, shows the effect of non-specific sensitization and its capacity to counteract the synaptic depression provoked by repeated stimulation. Figure 15 shows how the model predicts the dishabituation.

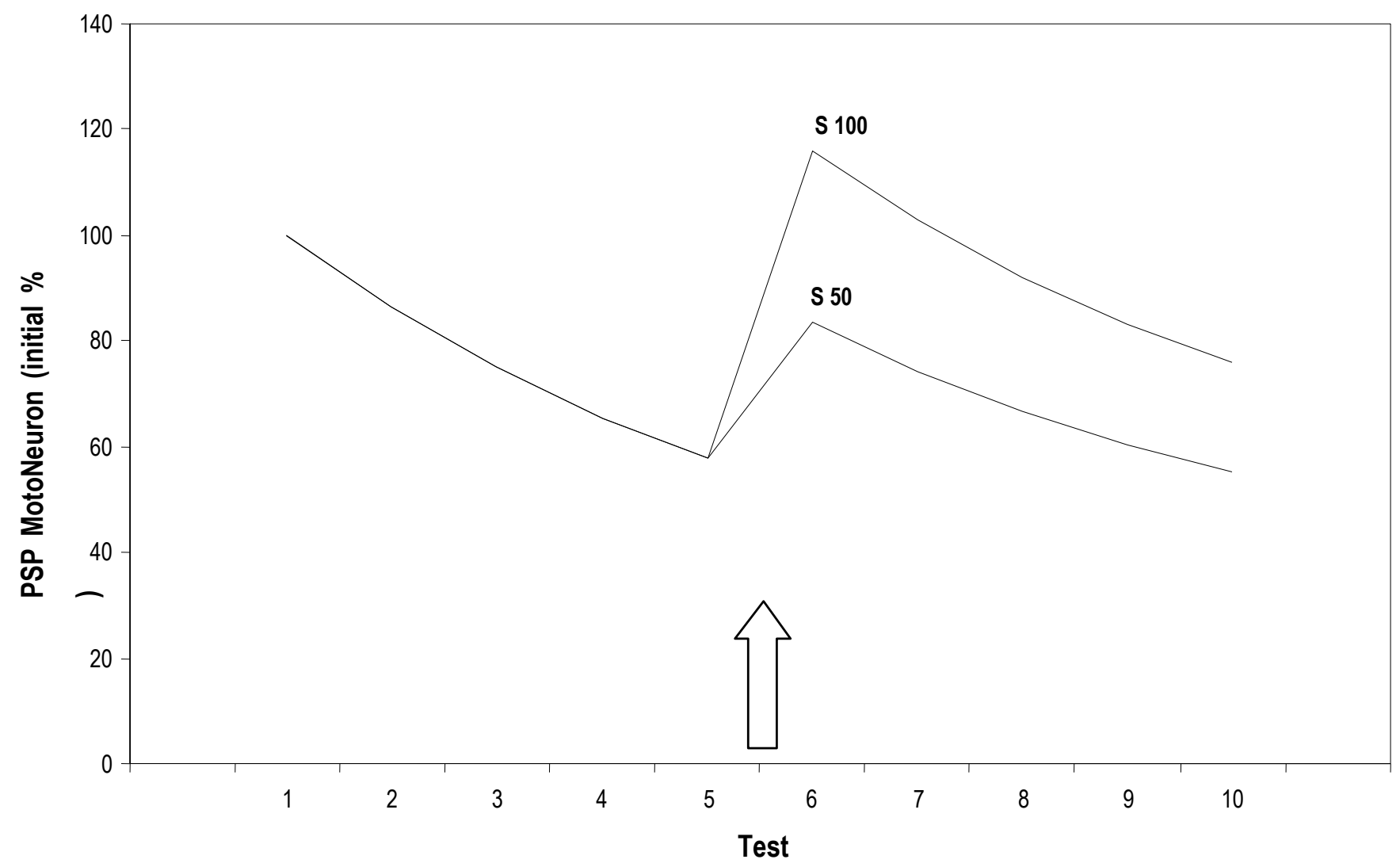

Figure 15. Dishabituation provoked by the administration of a sensitizing stimulus. While a series of stimuli is being administered (duration one cycle, intensity 1), the presentation of an intense stimulus (intensities of 50 to 100 arbitrary units), indicated by the vertical arrow, provokes the recovery of the synaptic efficiency depressed by the previous stimulation. After this dishabituating stimulus, there is still habituation of the previous stimulus with the same decrease rate.

\subsubsection{ASSOCLATIVE LEARNING}

Hawkin's model (1989a, b) predicts a great variety of phenomena related to classical conditioning. The following paragraphs present some of the most relevant phenomena, going from the most basic one to those that are considered of a superior order.

\subsubsection{Acquisition and extinction}

Figure 16 shows how the model adequately predicts both the acquisition of excitatory conditioning as well as its extinction, when the CS that was previously reinforced is presented alone. 


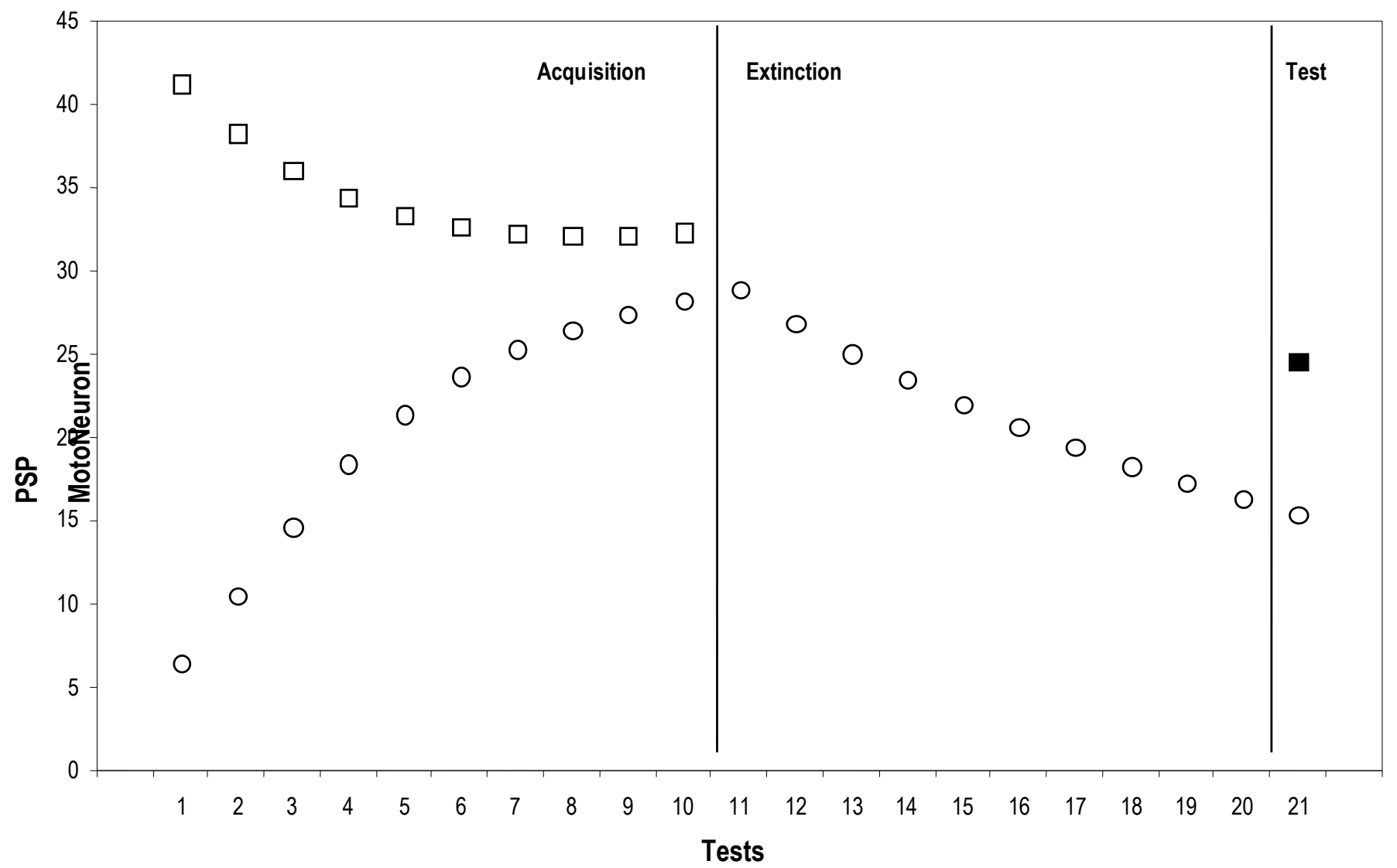

Figure 16. Acquisition and extinction of Pavlovian conditioning. Response of the motoneuron to the presentation of two stimuli: one CS (O), initially neutral, and one NS ( $\square$ ). The graphic shows two phases. The first phase consists of 10 reinforced tests, during which the CS gains the control of the response, whereas the NS loses it (i.e., it habituates). The second phase consists of 10 not-reinforced tests, during which the CS loses the associative force it previously acquired. Test 21 also shows the response of the motoneuron to the CS (a) of a control group (i.e., another Hawkins network) in which, during the tests of the second phase, no stimulus takes place (i.e., the same simulation cycles elapse, without administration of stimuli). In all the cases, CS as well as NS have a duration of one cycle (i.e., 10 seconds), whereas the CS has intensity 1, and NS has intensity 6.5 minutes elapse between each presentation of the CS.

\subsubsection{Conditions of experimental control}

This is one of the most relevant themes in the experimental analysis of behavior. It is assumed that from a behavioral point of view, the conditioned responses observed after a series of reinforced essays reflect some kind of association between the relevant events that occurred in each essay (i.e., stimuli and responses). If this is effectively so, an adequate control would consist of a procedure that does not allow the formation of such associations: each conditioning procedure that is supposed to be valid should generate a conditioned response level that is higher than this control. Several control procedures have been proposed in the course of time, all depending on the conditions that were considered necessary and sufficient for conditioning. Rescorla's proposal (1968) is generally considered to be the most adequate one-although it is also criticized for its random coupling of the NS and the CS. 
Anyway, a classic conditioning model should always show differential conditioning under these conditions, which is why we shall analyze the behavior of the Hawkins model in some of the most relevant control procedures.

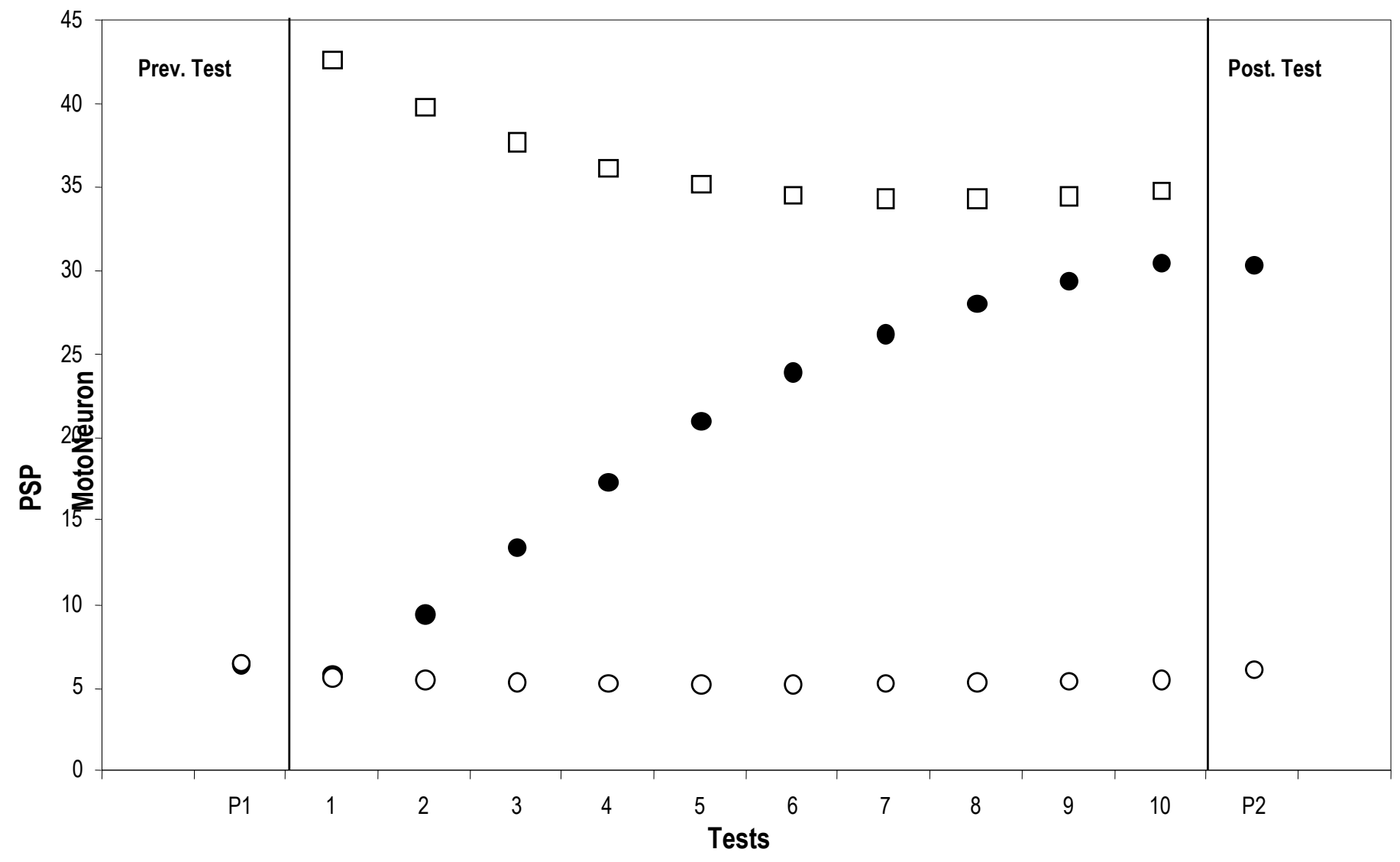

Figure 17. Differential Conditioning. Each of the 10 acquisition tests present three stimuli: CS1 (-), immediately followed by a NS ( $\square$ ), and CS2 (O), separated from the others by 15 cycles (i.e., the CS1 is being reinforced with an optimal interval, whereas the CS2 presents itself much before the NS, which means that its IBS >> optimal interval-is functionally speaking not reinforcing itself).

Figure 17 shows the results obtained by our computational model with a differential conditioning procedure, whereas figure 18 reflects the results obtained with more elementary procedures.

The differential conditioning procedure is very interesting, because the subject (i.e., the network) represents its own experimental control. Only the stimulus coupled with the NS with an optimal IBS increases its synaptic efficiency, and thus acquires the capacity to excite the motoneuron significantly. Figure 18 shows an usual relationship: $\quad$ Coupled $>$ NS-alone $>$ non-coupled $\approx$ non-trained $>$ CS-alone 


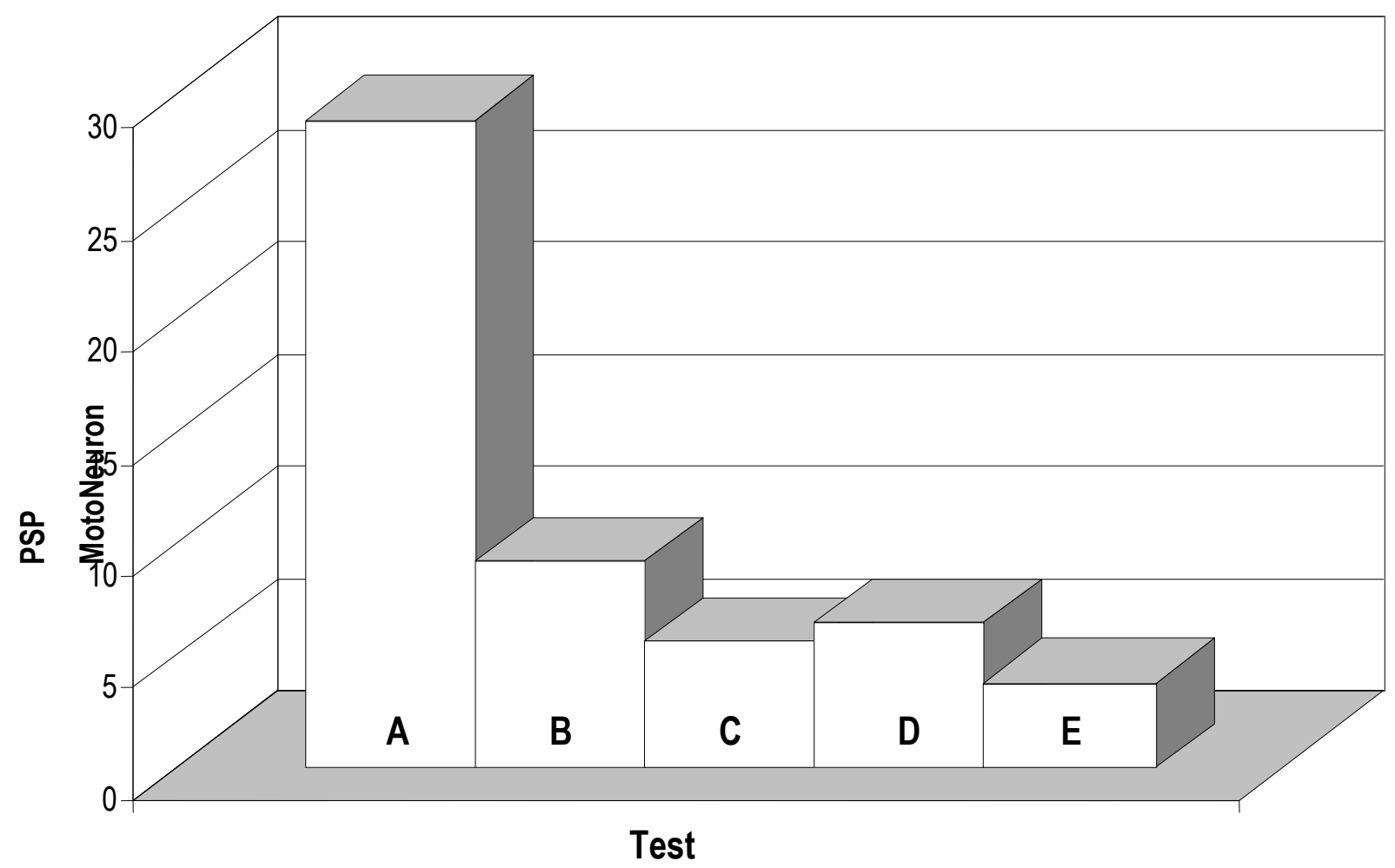

Figure 18. Various control procedures for the classical Pavlovian conditioning. In all the cases, we see the response of the motoneuron to the presentation of the CS during a test after the acquisition phase. A is the coupled procedure, in which the NS follows the CS with an optimal IBS (i.e., coupled condition; this procedure supposedly generates conditioning). During the acquisition tests of procedure B, only the NS presented itself (i.e., condition EI-alone). During the tests of procedure C both the CS and the NS are present, but not coupled (i.e., condition non-coupled; there are 20 cycles between CS and NS, in some tests the CS appears first, in others last). During the "acquisition" tests of procedure D not stimulus presents itself (i.e., non-trained condition). Finally, during procedure E only the CS is present (i.e., condition CS-alone). Whenever they were present, both CS and NS had a duration of 1 gycle, and an intensity of 1 and 6, respectively. Each test lasted about 5 minutes.

In the 'NS-alone' condition we observe the non-specific sensitizing effects derived form the presentation of the NS; in the 'not-coupled' condition, the effect of the non-specific sensitization is counteracted by the habituation that was provoked by the presentation of the CS, which will have similar effects to the 'non-trained' condition, according to the relation between the habituation rate and the sensitization rate; and finally, the 'CS-alone' condition shows the isolated effects of the habituation. All the previous effects appear in the coupled condition (i.e., habituation and non-specific sensitization), but the amplification of the sensitization, which depends on the much more powerful optimal coupling between NS and CS, is superposed on them.

\subsubsection{The effect of stimuli intensity}

Figure 19 and 20 respectively show the effect of the intensity of the CS and the NS. 


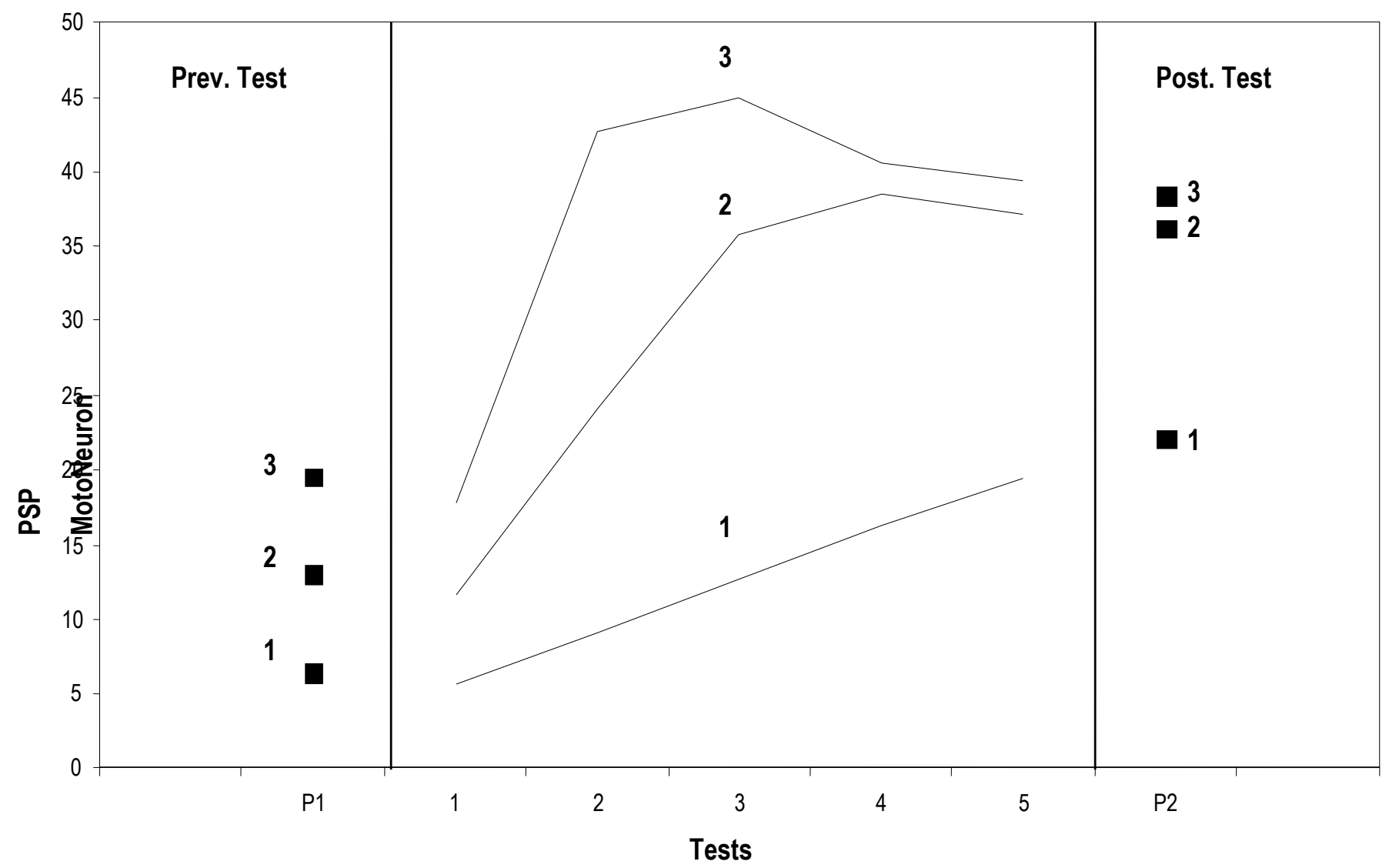

Figure 19. Effect of the intensity of the CS. The same conditioning procedure was administered to three networks (i.e., experimental groups), varying for each of them the intensity of the $C S(1,2$, and 3 arbitrary units). The procedure consists of 5 acquisition tests preceded and followed by an experimental one, as shown in the chart. The acquisition rate increases as the intensity of the CS increases. In all the cases, both CS and NS had a duration of $1 \mathrm{cycle}$, and the intensity of the NS was always 6 arbitrary units. Between two successive CSs, a period of 5 minutes elapsed.

We can observe that the intensity of the CS affects the acquisition rate. The shape of the curves shown in the graphic of figure 19 is closely related to the blocking process.

As in the previous case, figure 20 shows that a greater intensity of the NS leads to the observation of a greater level of conditioning.

In figure 21 we can observe a more thorough analysis, in which the intensities of both NS and CS are manipulated simultaneously. The relevant variable in this model is the relative intensity of both stimuli: The net difference in intensity determines the response level during the test after the conditioning phase and related to the values before this phase. 


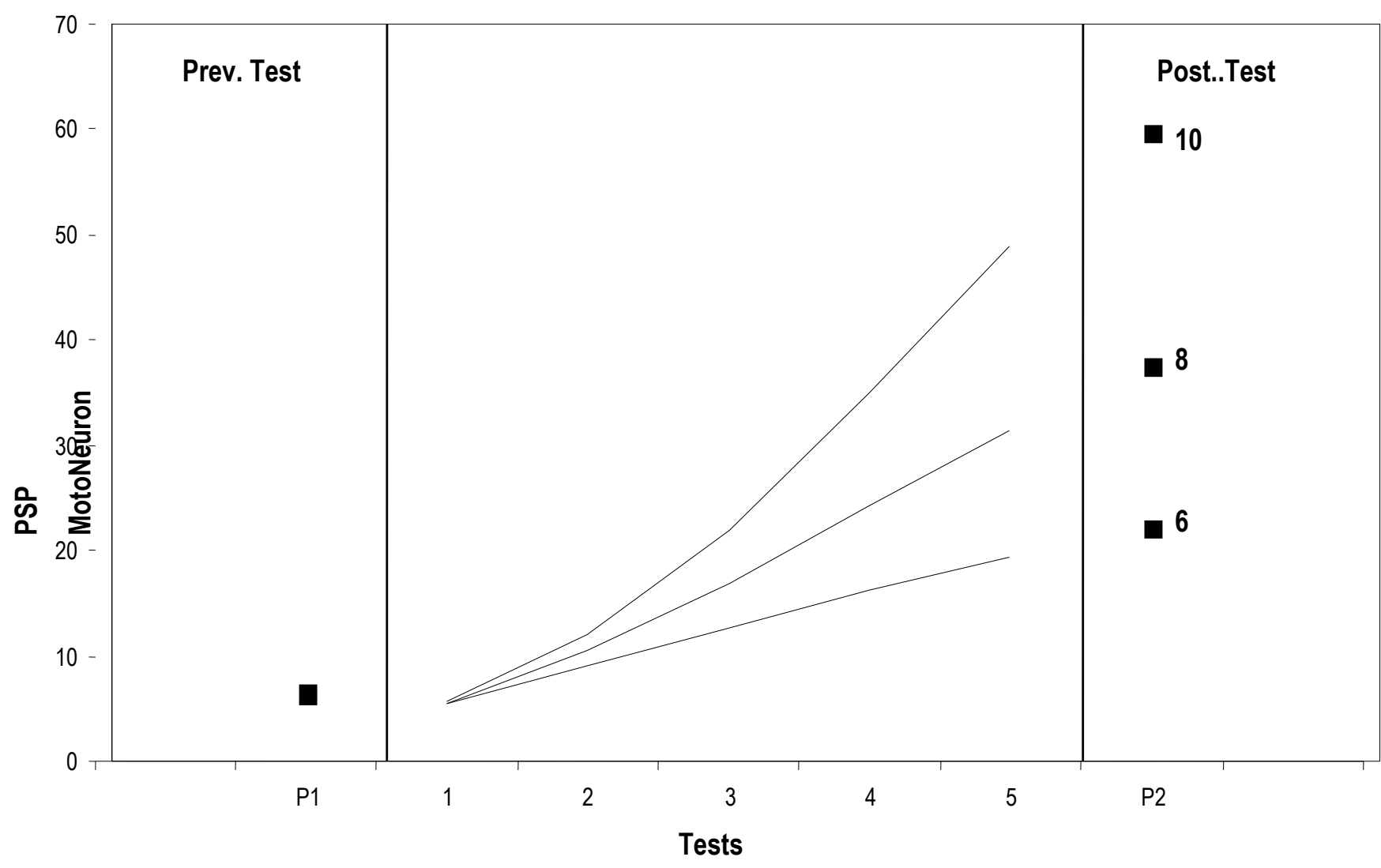

Figure 20. Effect of the intensity of the NS. The same procedures were administered to three networks (i.e., experimental groups), varying for each of them the intensity of the NS (the intensity of the CS being always 1). The stronger the intensity, the higher the conditioning level (bigher acquisition rate). The general conditions are similar to those of Figure 19.

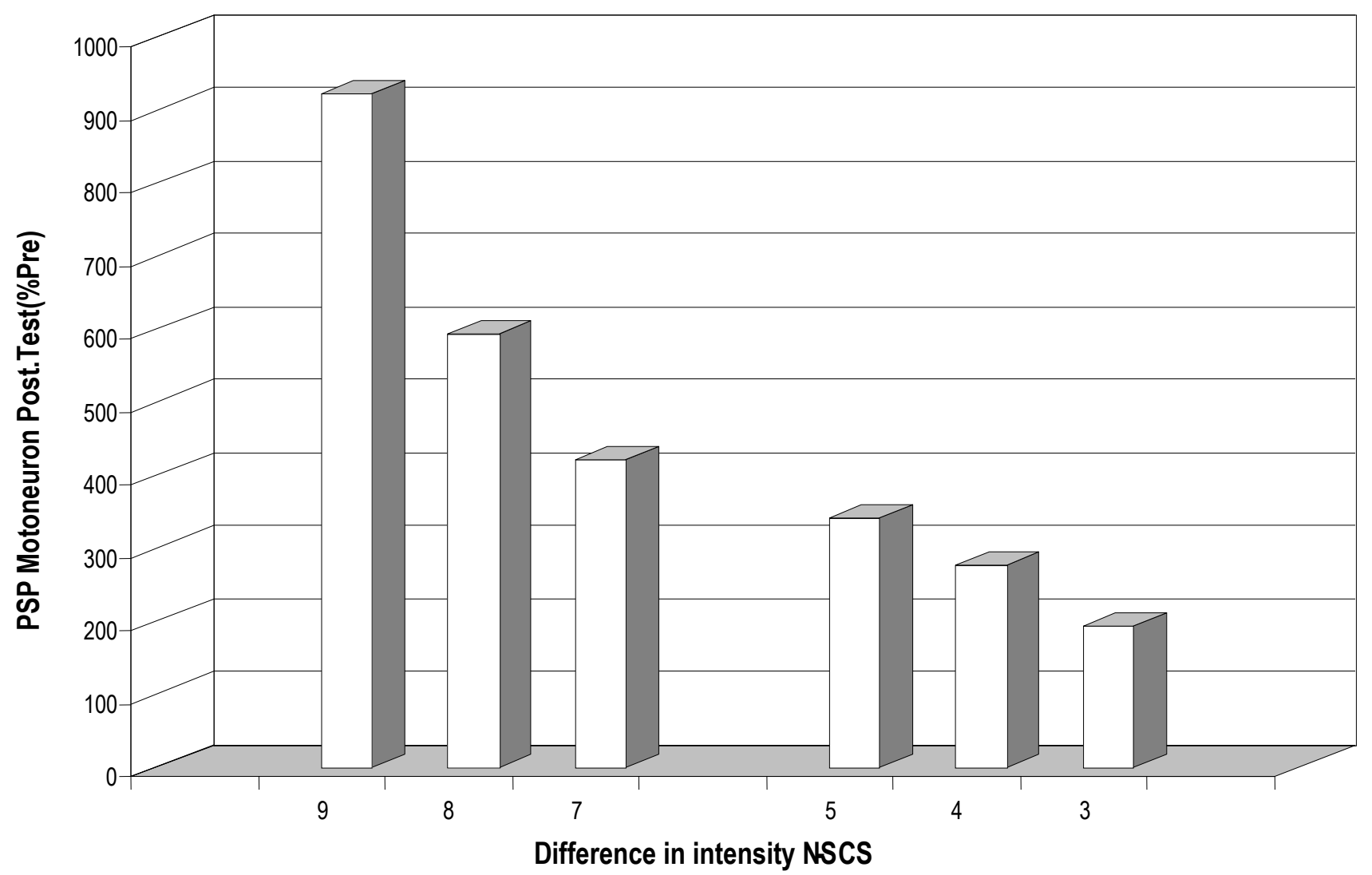

Figure 21. The difference in intensity CS-NS modulates the conditioning level that can be generated in an acquisition procedure. In this case, the intensity of the NS (6 or 10 arbitrary units) as the CS (1, 2, or 3 
units) varied simultaneously, administering each of the different combinations to 6 networks (i.e., experimental groups). The chart shows the response of the motoneuron during the post-acquisition experiment as a percentage of the response given during the pre-acquisition experiment.

\subsubsection{The effect of the interval between tests}

Generally, a larger interval between tests leads to a greater acquisition level (i.e., the distributed practice is more effective than the massive practice). The implementation can model the interval between tests in two ways: By creating an empty test between two conditioning tests (i.e., conceptually closer to what is usually understood by 'interval between tests'), or by doing conditioning tests that are consecutive but longer (i.e., including simulation cycles before and $\mid$ or after the stimuli that appear). Since both methods are functionally identical from the point of view of the stimulation, the second approach is used (i.e., an interval of 5 minutes between intervals implies 30 cycles of simulation between two consecutive CSs).

Figure 22 shows the effect of the interval between tests, understood as time between stimulation.

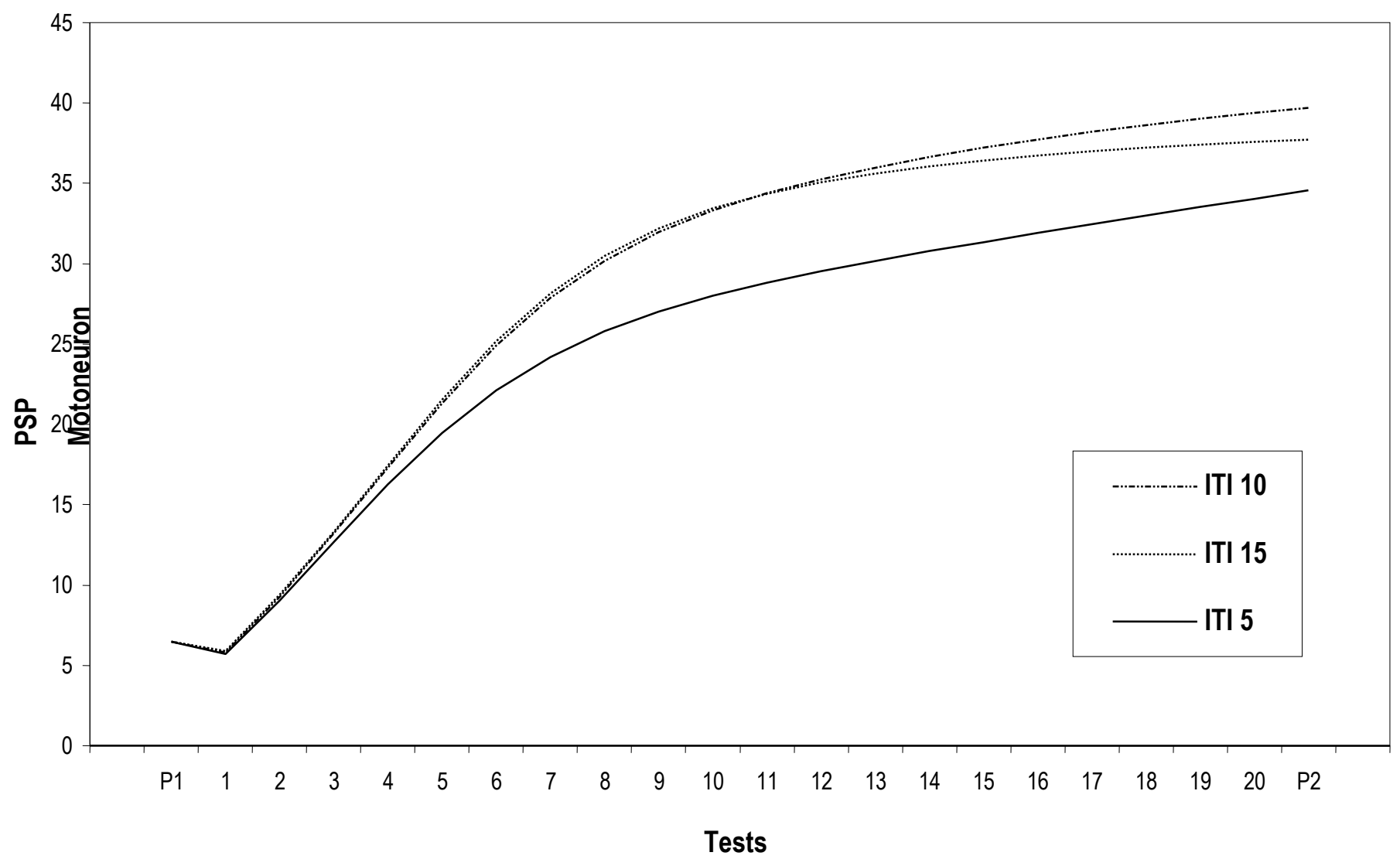

Figure 22. Effect of the interval between tests. The interval (ITI: Inter Trial Interval) is expressed in minutes.

\subsubsection{Temporal relations NS-CS}

The Hawkins model simulates in an elementary way the interval between stimuli (i.e., SOA) by using a temporal window -usually with the amplitude of one cycle- to determine the eligibility state of a synapse. In this 
paragraph we shall consider not only the starting moment of both NS and CS (i.e., the parameters to define the SOA), but also their finishing moment. This will allow us to analyze the efficiency of the diverging conditioning procedures, and in particular of two trace procedures -one of them with a trace interval of one cycle, the other with a trace interval that is zero-, as well as a short delay procedure.

When Hawkins (1989a, b) presented his model, he argued that a delay procedure is more efficient than a trace procedure, which would be equivalent on other occasions (i.e., his model behaves like the animal experimental subjects). This is due to the fact that during the delay procedure the CS and the NS end up by overlapping each other, and thus adding up their effects to the facilitator interneuron, wich means that, a priori, stronger sensitization signals will be generated and consequently a greater presynaptic facilitation will occur. However, this is only partially correct, because Hawkins does not take into account the accommodation of the interneuron.

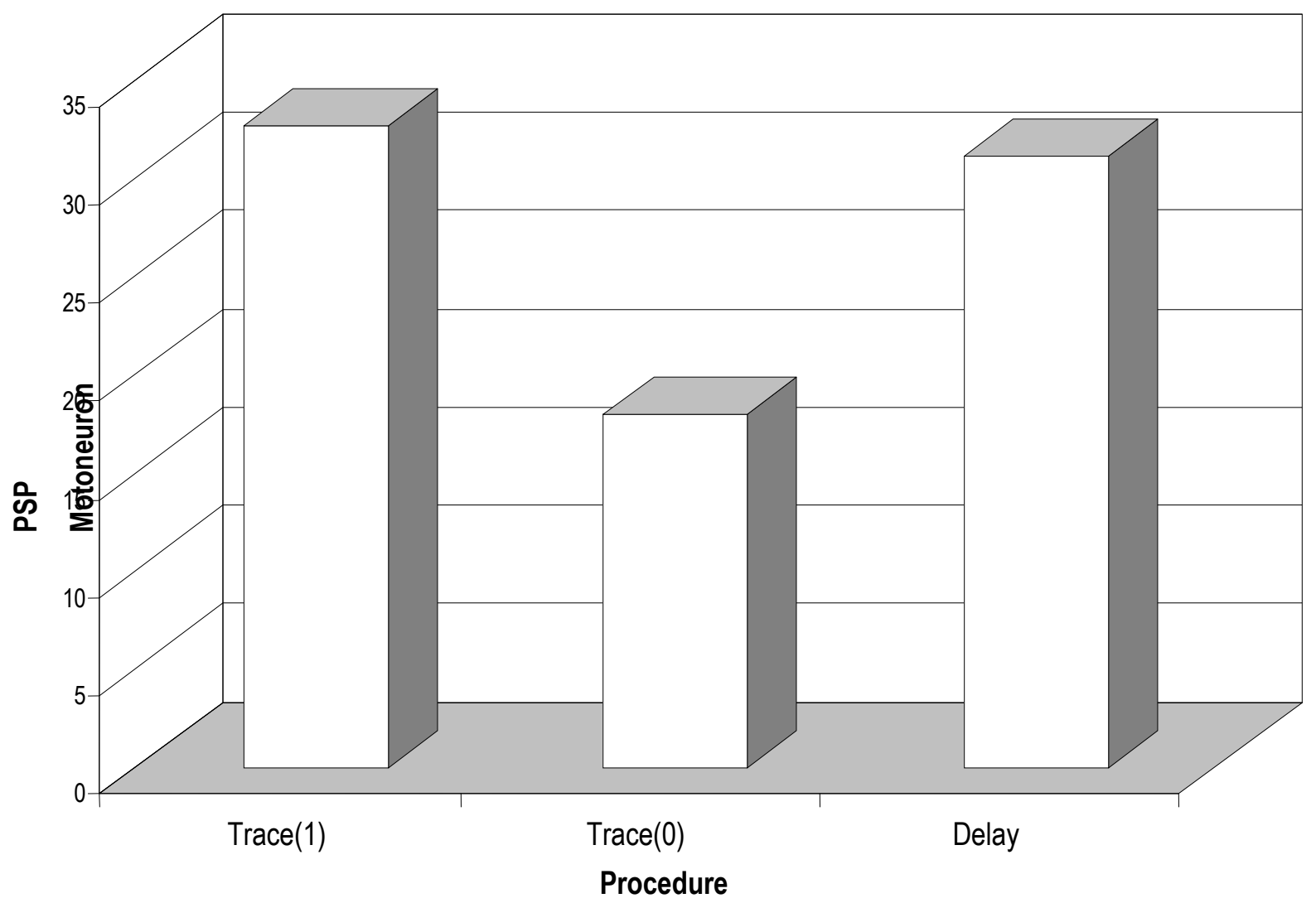

Figure 23. Comparison of two trace procedures and a short delay procedure. The graphic shows the response of the motoneuron during the test after the acquisition phase.

Figure 23 allows us to compare the efficiency of these procedures. The delay procedure is more efficient than the trace procedure if the applied trace interval is minimal (i.e., zero cycles: the NS starts when the CS finishes). However, with a larger trace interval -in this case, a cycle-, the delay procedure turns out to be slightly worse. Hawkins (1989a, b) affirms that when NS and CS overlap during the delay procedure, the interneuron receives a 
greater stimulation level, which increases the value of its PSP (Postsynaptic Potential). Nevertheless, the sensitization signal generated by the interneuron ( $\mathrm{S}$ in the model) will depend on the value of the activation in the immediately preceding cycle (i.e., it depends on the threshold $\mathrm{T}: \mathrm{S}(\mathrm{t})=\mathrm{PSP}(\mathrm{t})-\mathrm{T}(\mathrm{t})$, with $\mathrm{T}(\mathrm{t})=\mathrm{PSP}(\mathrm{t}-1)$; or also: $\mathrm{S}(\mathrm{t})=\operatorname{PSP}(\mathrm{t})-\operatorname{PSP}(\mathrm{t}-1))$. During the delay procedure, the activation that results from the united presentation CS-NS, PSP $(\mathrm{t})$ will have to exceed the activation generated during the previous cycle by the CS, $\operatorname{PSP}(t-1)$, in order to produce a $\mathrm{S}(\mathrm{t})>0$ value. This means that the additive effect (wich made Hawkins affirm that the delay procedure is superior to the trace procedure) will be reduced by the associative force of the CS: In practical terms it will be equivalent to the contribution of the NS (which is much smaller, given that due to the habituation process, the efficiency of the CS in the t-cycle is smaller than the one it had in the $t-1$ cycle). During the trace procedure with a zero trace interval, $\operatorname{PSP}(\mathrm{t})-\mathrm{t}$ being the moment on which the NS presents itself depends only on the NS and $\operatorname{PSP}(\mathrm{t}-1)$ will be provoked, as in the previous case, by the last CS cycle: $\mathrm{S}(\mathrm{t})$ will be smaller than in the delay case, because $\operatorname{PSP}(\mathrm{t})$ is also smaller. However, when we consider a trace procedure with a trace interval above zero, the results are remarkably different. If we establish once again $t$ at the moment when the NS is administered, we may see that $\mathrm{S}(\mathrm{t})$ equals $\operatorname{PSP}(\mathrm{t}) ; \operatorname{PSP}(\mathrm{t}-1)$ is zero, because there is no stimulation during the trace interval. Now the sensitization signal $\mathrm{S}(\mathrm{t})$ will have a value that is slightly superior to the one it has during the delay procedure, and will therefore generate higher conditioning levels, as shown in figure 23.

\subsubsection{Conditioning of the second order}

One of the fundamental functions of the interneuron is the distribution of sensitization signals, controlled by any kind of stimulus which is able to activate it. A direct consequence of this fact is the possibility to generate conditioning of a higher order in which the CS acts as a reinforcer (i.e., conditioned reinforcer). Figure 24 shows how the computational implementation of the Hawkins model (1989a, b) adequately predicts the conditioning of the second order.

\subsubsection{Shadowing}

The phsysical characteristics of an element in a stimuli set not only determine the associative force acquired by this element, but also the force acquired by the other elements of the set, generally reducing it. This phenomenon of competition among stimuli is generally known as shadowing: The elements mutually 'eclipse' each other. Figure 25 shows how the computational implementation of the Hawkins model (1989a, b) predicts this phenomenon. 


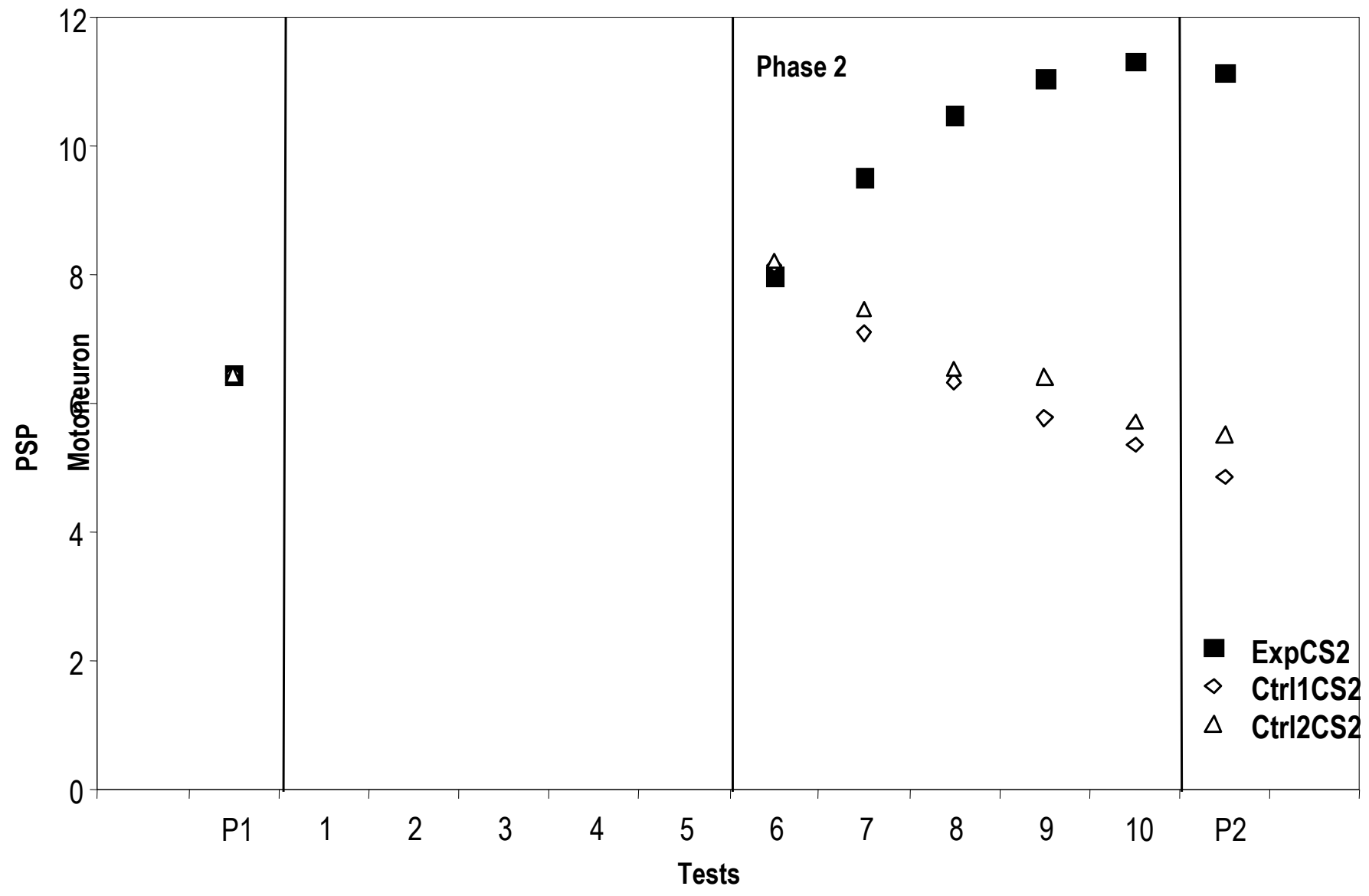

Figure 24. Conditioning of the second order. The procedure includes three networks (i.e., three experimental groups), of which two function as control ones, and it is divided into two phases. The Experimental group receives 5 reinforced $C S_{1}-N S$ tests during the first phase. The second phase of this group consists of the administration of another 5 tests during which a new $C S_{2}$ and the $C S_{1}$, of the previous phase, which functions as a reinforcer, are being coupled, with an optimal interval. The Controllgroup receives five tests during the first phase, during which the same EC $C_{1}$ and EI as in the previous group are presented, but not coupled (i.e., with an interval that is much bigger than the optimal one: 15 cycles). The second phase is identical to the one of the Experimental group. The group Control2 has a first phase that is identical to that of the Experimental group; however, during the 5 tests of the second phase, $C S_{2}$ and $C S_{1}$ appear as non-coupled. Only the response of the motoneuron to the stimulus, added in the second phase, is shown. In all the cases, the stimuli have a duration cycle, and an intensity 1 for the CSs and 6 for the NS.

\subsubsection{Blocking}

Interstimulative competition can be generated according to the differences in conditioning level (or conditioned shadowing) that each element of the stimulative compound reaches independently. Figure 26 shows an example of this. In this case, the conditioning level of one of the compound elements, CS2, depends on the conditioning history independently from the other element, CS1.

Blocking occurs when a stimulative element, that is pretrained in isolation, reduces the capacity to acquire associative strength of other stimuli when it forms a stimulative compound with them. 


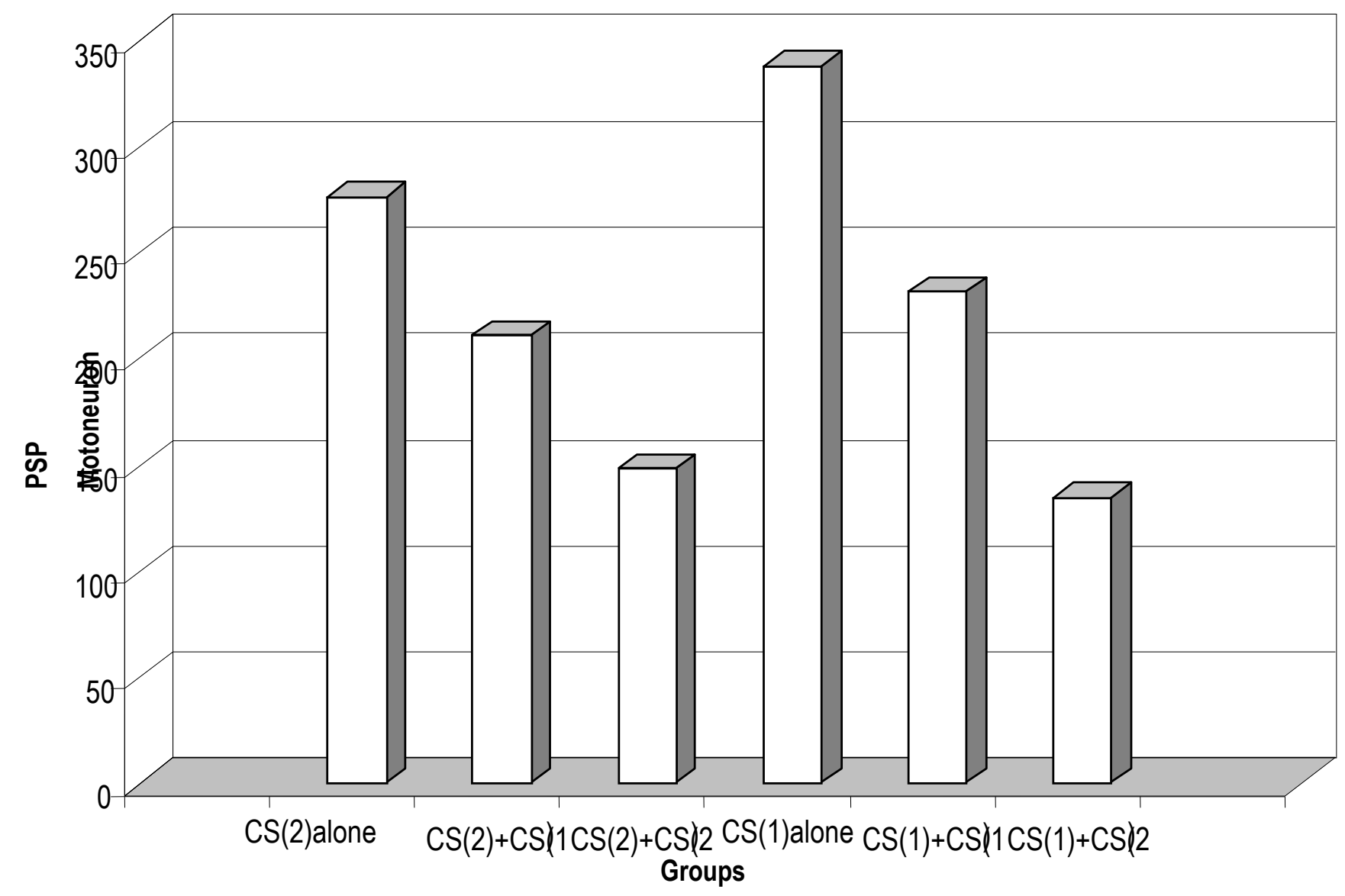

Figure 25. Shadowing according to the stimulation intensity. The procedure consists of 5 reinforced tests of a stimulative compound CS(i)+CS(i) (i.e., both stimuli start and end at the same time, the value between brackets indicates the intensity of the stimulus). For these six different groups (i.e. networks), the intensity of both CSs was manipulated by combining the values 0, 1 and 2 arbitrary units (e.g., the group CS(2)Alone implies the administration of a CS with intensity 2 and another with intensity 0 -is not present: This is a control condition). We can observe the existence of mutual darkening, which increases as the stimuli's intensity increases. In all the cases, the intensity of the NS was 6 units, and the interval between the presentations of the stimulative compound was 5 minutes. The graphic shows the response of the motoneuron in a test carried out after the acquisition phase, as a percentage of the response given during a previous test.

In their model, Gluck and Thompson (1987) expected the facilitator interneuron to be sensitive to the stimulative conditions that usually generate blocking, but unfortunately their model is not able to generate it. However, the computational model of Hawkins (1989a, b) does predict this highly relevant phenomenon, as can be seen in figure 27.

Figure 28 shows how blocking evolves in the course of the tests of the standard procedure's second phase. The second test produces a higher blocking level. 


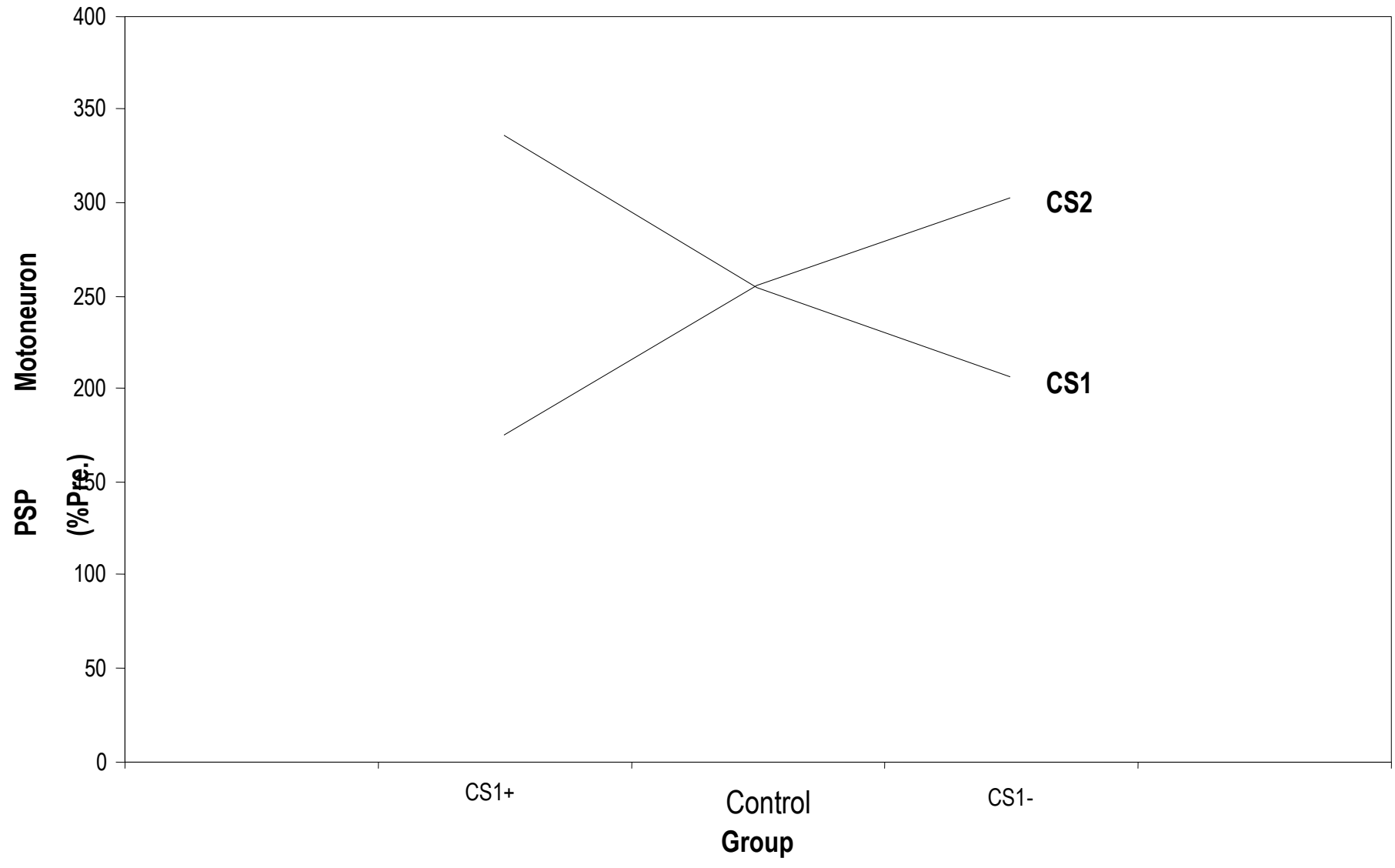

Figure 26. Conditioned Shadowing. The procedure includes three groups (i.e., three different networks). All three receive an acquisition phase formed by reinforced tests $\left(C S_{1}+C S_{2}\right)-N S$ (i.e., a stimulative compound such as CS appears). For each group, these reinforced tests alternate with extra tests (i.e. 9 tests in total, 5 standard and 4 extra tests, starting and ending with standard ones). In the CS + group, the extra tests consist of the reinforced presentation of the $C S_{1}$ (i.e., the same as the stimulative compound of the standard tests). In the CS 1 -group, extra tests are administered which consist of the non-reinforced presentation of the $C S_{1}$ (i.e., it appears alone). In the control group, the extra tests do not include stimuli (i.e., an equivalent time elapses). The graphic shows the response of the motoneuron to the $C S_{2}$ in a test after the acquisition, represented as a percentage of the response that was given before the start of the conditioning (i.e., in a pre-acquisition test). In all the cases, the stimuli have a duration cycle and an intensity 1 for the CSs and 6 for the NS.

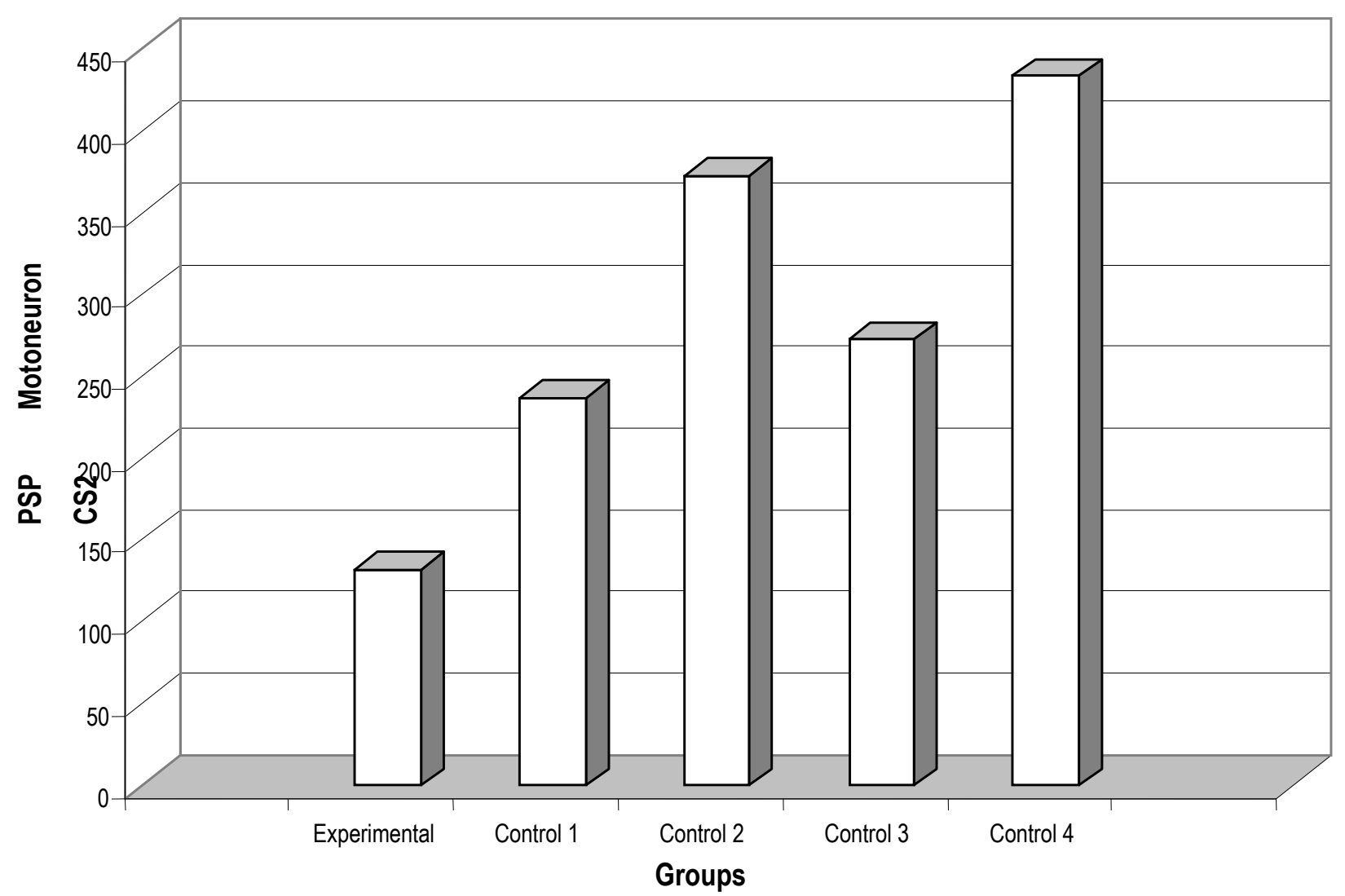


Figure 27. Blocking. The procedure includes 5 different groups (i.e., five networks), four of which function as controls. Each blocking procedure includes two phases. The group Experimental receives reinforced tests CS $1-N S$ during the first phase, with an optimal interval; during the second phase, a $C S_{2}$ is added which forms a stimulative compound with the pretrained $C S_{1}$, and this compound is continuously reinforced during several more tests (i.e., tests $\left[C S_{1}+C S_{2}\right]-N S$ ). The group Control1 receives the same number of tests during the first phase, although no stimulus is present (i.e., the same simulation time elapses); the second phase is identical to that of the Experimental group. The group Control 2 has a first phase which is identical to the Experimental phase, but during the tests of the second phase the $C S_{1}$ does not appear (i.e., $C S_{2}$ is replaced by $C S_{1}$ ). The group Control3 receives during the first phase the same stimuli as the group Experimental, but in this case, not coupled (i.e., there is a SOA of 15 cycles between both stimuli); the second phase is identical to that of the Experimental group. Finally, the group Control4 only differs form the group Experimental in the content of the second phase: the added $C S_{2}$ is reinforced by the NS but it does not form a stimulative compound with the $C S_{1}$ (i.e., $C S_{1} \nrightarrow\left[C S_{2}-N S\right]$ ). It is said that there is blocking because the conditioning level of the $C S_{2}$ in the group Experimental is inferior to that of any of the control groups. The results show the response of the motoneuron to the CS 2 in a test after the second phase, expressed as a percentage of what the same stimulus generated in a test before the conditioning procedure. In all the cases, the duration of the stimuli is of one cycle; the intensity is 1 for the CSs and 6 for the NS. The tests bave 32 cycles (i.e., about 5 minutes).

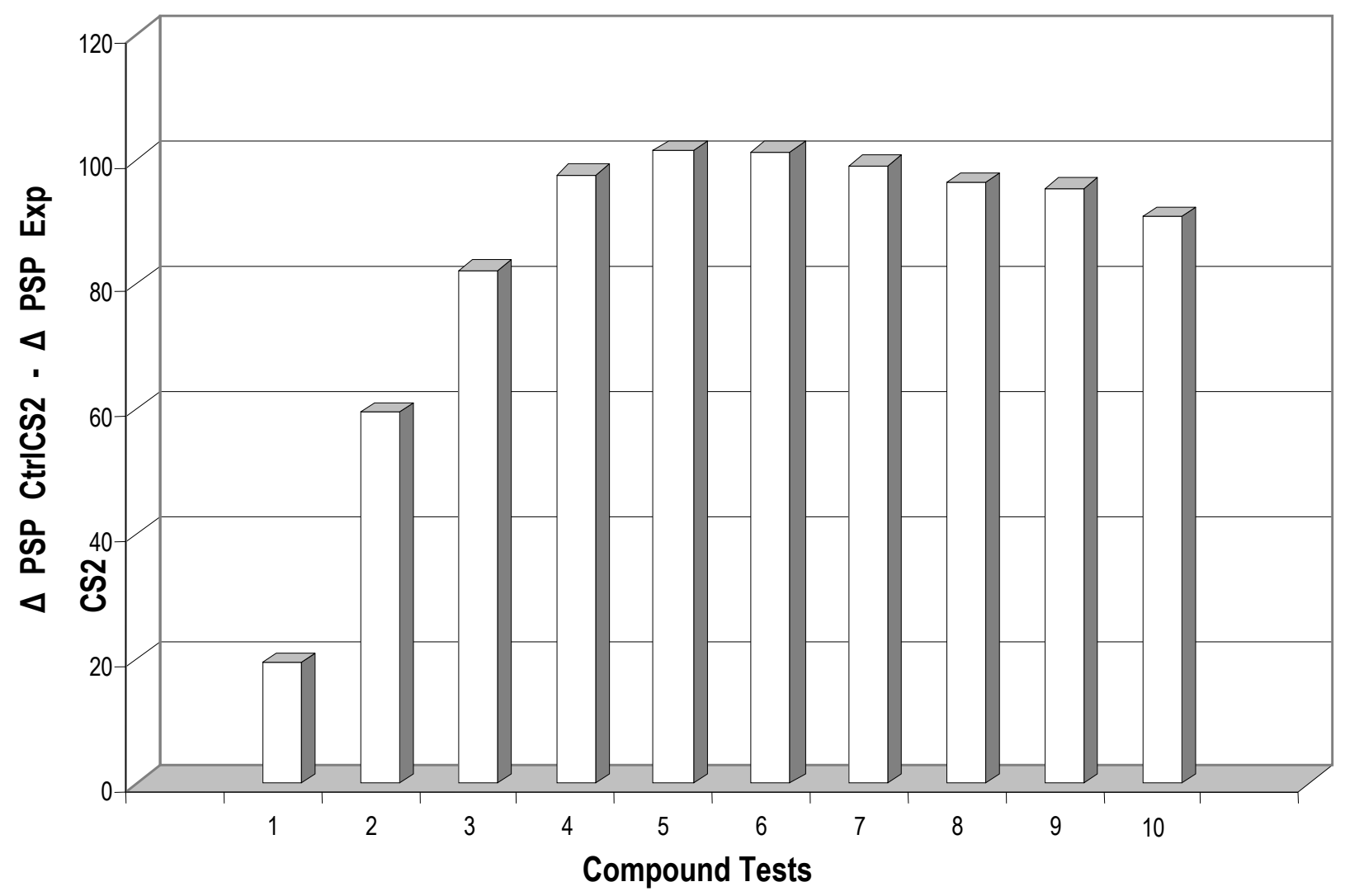

Figure 28. Evolution of the blocking in the course of the compound tests of the second phase of the standard procedure. A total of 20 different networks was used (i.e., experimental groups), to which 1 to 10 compound tests were administered during the second phase, coupled in 10 couples of two groups: an experimental group (i.e., it receives reinforced tests during the first phase), and a control group (i.e., it does not receive stimuli during the tests of the first phase). The results are shown as the difference between the control group and the experimental one in the increase of the associative force of the stimulus added during the second phase (i.e., a high value indicates a stronger blocking); the response level during the test after the conditioning is shown as a percentage of the response given in a test before the conditioning. 


\subsubsection{Contingency CS-NS and conditioning}

In the previous paragraph we explained how the Hawkins model (1989a, b) adequately predicts the basic aspects of blocking; figure 29 shows how it predicts variations in the conditioning level that accompany the variations of the CS-NS at contingency level: As the predictive power of the CS decreases, so does the conditioning level.

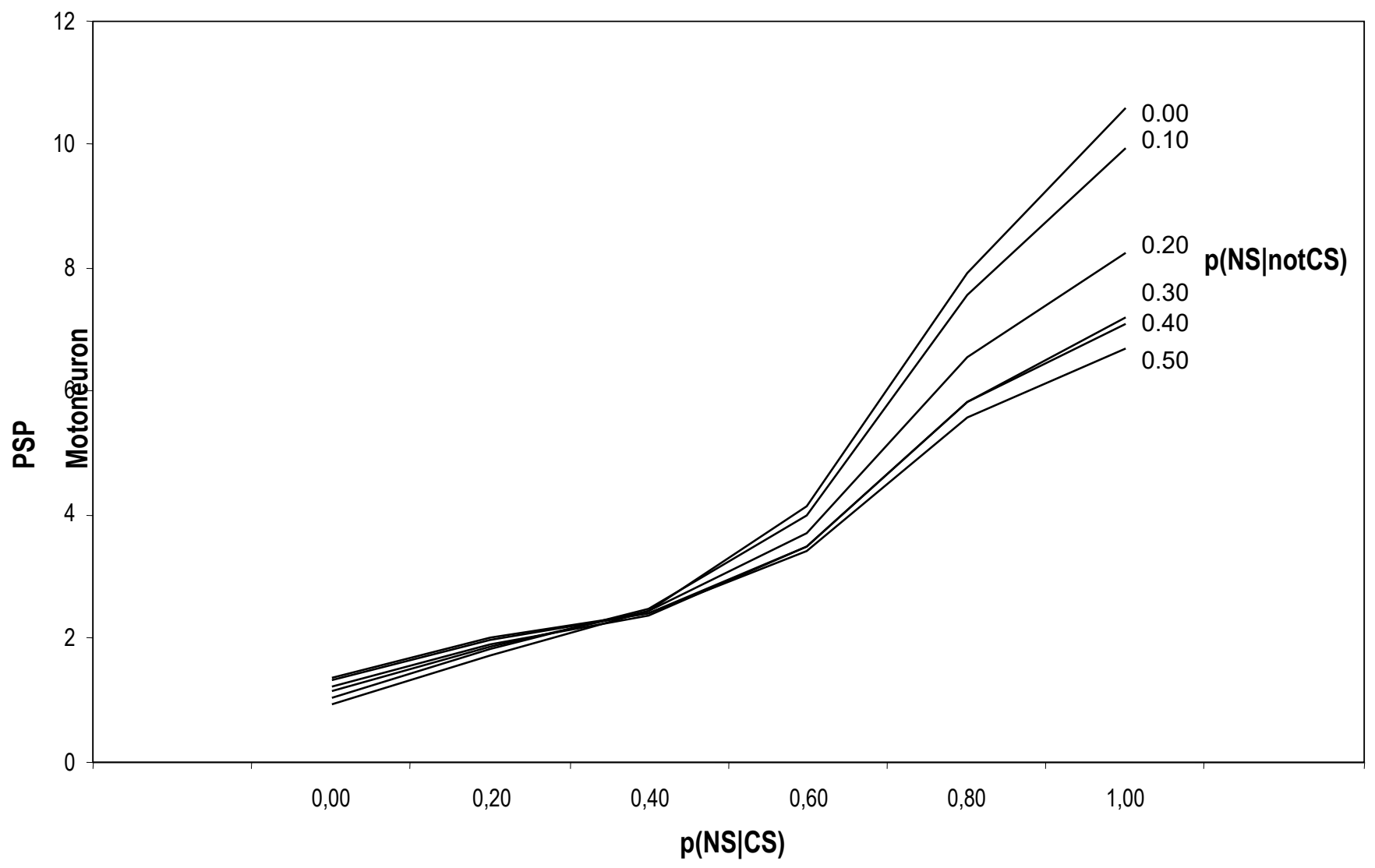

Figure 29. Effect of the CS-NS contingency. Defined as the relationship between $p(N S \mid C S)$ and $p$ (NS $\mid$ notCS), the contingency modulates the conditioning level that can be reached in a determined situation. The chart shows the results obtained with a very particular procedure, which generates low conditioning levels -which explains the values of the motoneuron. Even so, we can see the influence of the contingency. More specifically, we created a series of 30 tests, with 3 cycles for each test. The basic configuration consists of two types of tests: one that includes a CS of two cycles, and another one that does not contain stimuli (i.e., this configuration corresponds to the relationship $p(N S \mid C S)=0$, $p(N S \mid$ notCS $)=0)$. Several contingency conditions were created with this configuration, , adding NSs at random. The final result consists of 36 stimuli series, derived from the combination of the values 0.0, 0.2, 0.4, 0.6, 0.8, and 1.0 for $p(N S \mid C S)$, and 0.0, 0.1, 0.2, 0.3, 0.4, and 0.5 for $p(N S \mid$ notCS). The results depend on the final distribution of the stimuli in the course of the 90 simulation gycles of each series, but they allow global comparisons. In general, the response level of the motoneuron decreases with values that are smaller than $p(N S \mid C S)$ (i.e., the amount of reinforced $C S s$ decreases). For a given value of $p(N S \mid C S)$, the conditioning level decreases as $p(N S \mid$ notCS) increases (i.e., the amount of NSs that are not preceded by a CS). Globally speaking, the conditioning level decreases together with the predictive force of the CS.

According to the results of figure 29, the effects of the contingency depend on the habituation suffered both by CS and NS when they are presented alone. However, an alternative and more realistic explanation is based on the blocking phenomenon. If we assume that every single stimulus of a conditioning procedure takes place on a heterogeneous basis of stimuli (i.e., the stimulative context), we can also assume that, in essence, each conditioning procedure can be reduced to a situation of stimulative competition, not only between the explicitely 
manipulated stimuli, but also between the latter and the stimulative context. In that respect, a reinforced CS-NS test would be actually a test of the [CS+Context]-EI type; while a test with a 'single' NS would be actually a reinforced Context-NS test (e.g., in the $\mathrm{p}(\mathrm{NS} \mid$ noCS $)=1.0$ condition, all the tests reinforce the context). In this test, the context would acquire an associative force that would allow it to darken, albeit slightly, any CS with which it forms a stimulative compound in ulterior tests. A 'single' CS test would be actually a [CS+Context]notNS test, in which both the CS and the Context would lose associative power.

We repeated the procedure shown in figure 29 in order to explore this alternative, but added a $\mathrm{CS}_{2}$, which is present in all of the cycles, to simulate in a very elementary way the stimuli Context. Figure 30 summarizes the results, which are visibly equivalent.

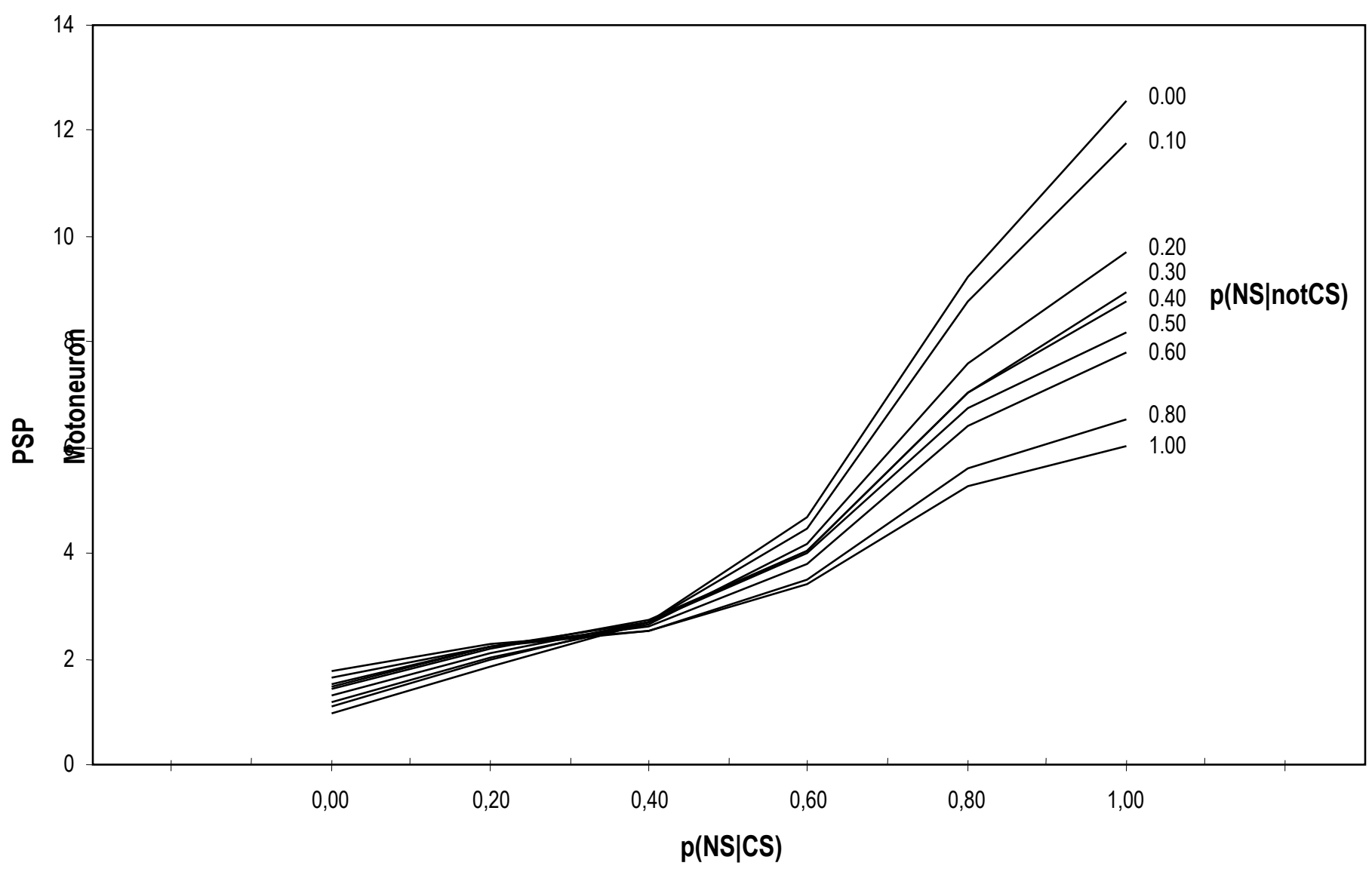

Figure 30. Effects of the contingency when the stimulative context is explicitly introduced. The procedure is identical to the one described in the Figure 29, but it includes a second $\mathrm{CS}_{2}$ that is present in all the simulation cycles. The range of values for p(NS| notCS) was also amplified so that 54 series of stimuli can be administered independently to a Hawkins network.

Figure 31 shows the conditioning of the context in each condition. The procedure we apply implies a massive administration of stimuli in a short lapse of time (15 minutes of simulation time), which improves the habituation and damages the conditioning process. As a result, we can use the usual procedure to define 
$\mathrm{p}(\mathrm{NS} \mid$ notCS $)$ by dividing the time in which CS is absent in periods that have the same duration as the CS. We would also have to use a long CS in order to implement a long stimulation session, which would accelerate the habituation. In spite of its limitations, this procedure allows us to test the effects of the contingency on the conditioning level.

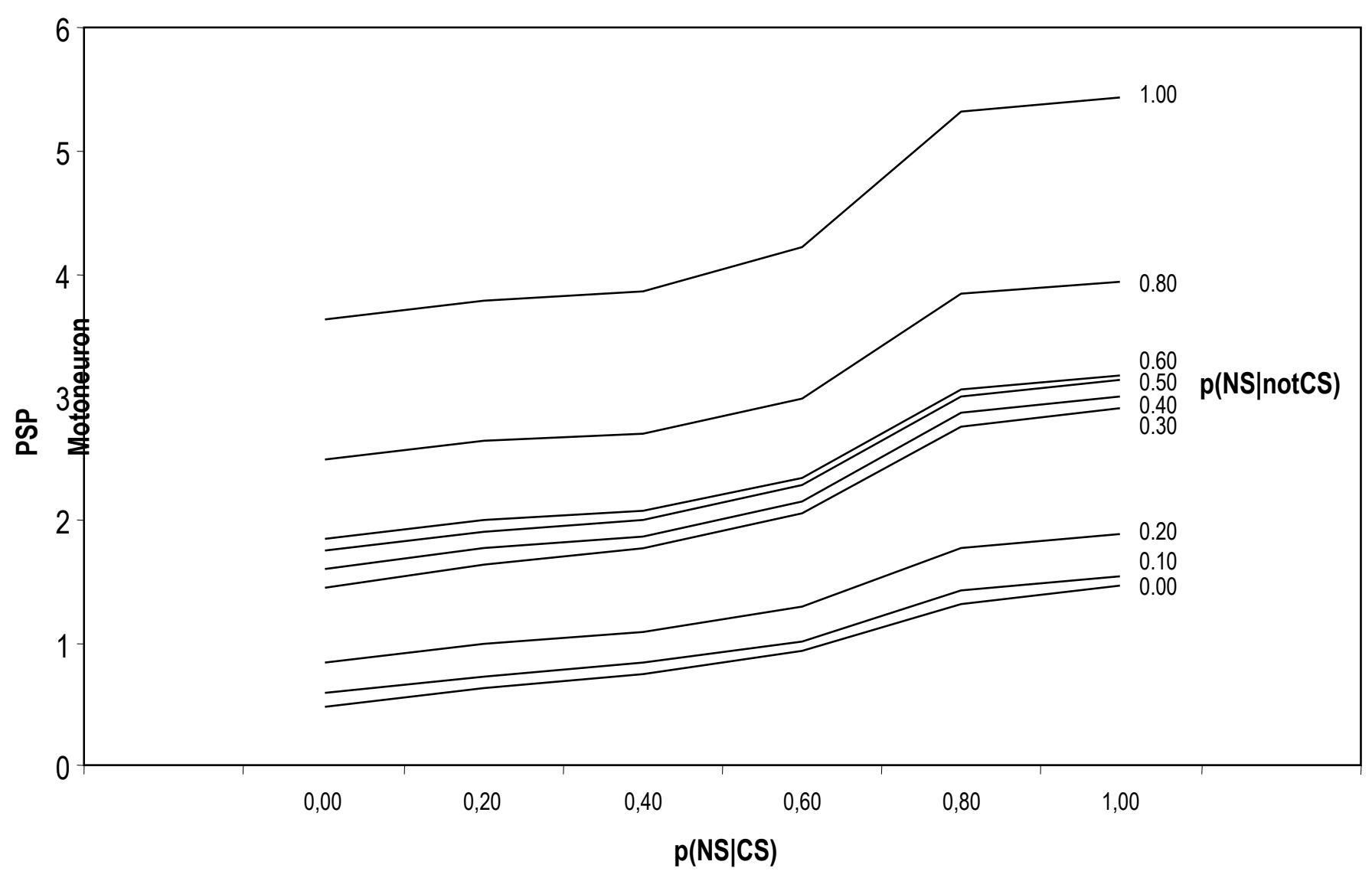

Figure 31. Conditioning of the stimulative context in the procedure applied in Figure 30.

\subsubsection{The effect of the pre-exposure of CS and NS}

This manipulation is related to the previous paragraph and it includes a phase in which the CS or the NS are presented alone, several times, before the start of the conditioning phase. Figure 32 shows the effects of both manipulations.

In both cases, the effect of pre-exposure (which implies a delay of the ulterior acquisition) can be explained by the habituation of the pre-exposed stimulus. 

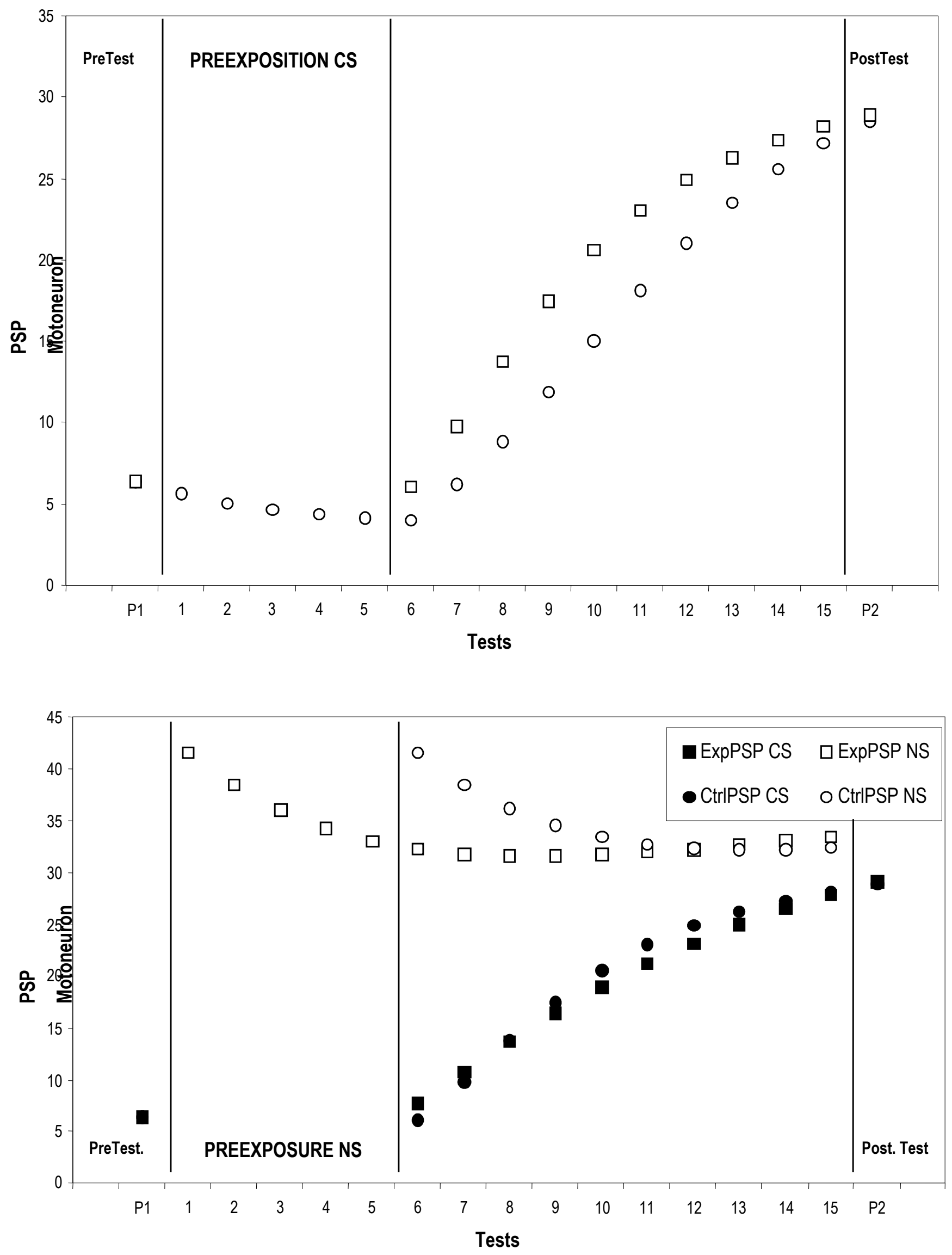

Figure 32. Effect of the pre-exposure to the CS (upper chart), and to the NS (lower chart). In both cases, the acquisition phase, formed by 10 reinforced CS-NS tests, is preceded by a pre-exposure phase that includes 5 isolated presentations of the CS or the NS. 


\section{CONCLUSIONS}

Certain branches of Psychology, Biology and the Computer Sciences focus on the study of the learning process, claiming that the essential aspects of this phenomenon are their exclusive field of competence. However, we must try to search for converging results by showing the multiple aspects of one reality.

The Artificial Intelligence paradigm called ANN or Connectionist Systems leaves room for this convergence and in fact represents a decisive turn towards the biological and psychological models. The ANNs use the structural and functional properties of biological systems to implement learning laws that are derived form the behavioral analysis of these systems. They create systems that consist of a certain number of processing units which have extremely simple computing capacities and are interconnected more or less profusely and according to several patterns. The dependence stimulus-response, generally imposed by the environment, is defined through a function that makes certain parameters (i.e., variables) depend on this interconnected set and on the stimulative conditions with which it coexists (i.e., the environment); a subset of these parameters is seen as the response returned to the environment by the system. The level of convergence with Biology provides the processing units and their interconnections pattern with a determined neurophysiological plausibility, turning them from an arbitrary data structure into Artificial Neural Networks. The level of convergence with Psychology makes it possible for the parametrical modification rule to transcend the ad hoc algorithm level and to become a learning rule.

This article has analyzed two restrictive ANN models and studied their differences and similarities. Table 1 summarizes the most significant differences:

The results of the two models diverge considerably. The $\mathrm{GT}_{\mathrm{m}}$ covers a small part of the simple learning phenomena detected in Aplysia. One of the phenomena that directed the specification of its equations, blocking, is remarkably absent: The behavior of the circuit does not adjust to the behavioral data or models on which it seemed to be based. Strangely enough, the authors assume that this is a defect of the $\mathrm{HK}_{\mathrm{m}}$ and not of their interpretation of it. It is important to remember that the $\mathrm{HK}_{\mathrm{m}}$ may be entirely incorrect, even though it is based on a rigorous neurobiological analysis -its authors define a minimal circuit by chosing a small part of the neural machinery that is relevant for the behavioral study of Aplysia, and this choice is influenced by criteria derived 
form the behavioral models. Even so, the $\mathrm{HK}_{\mathrm{m}}$ makes a generic proposal, whereas the GT $\mathrm{m}$ creates a set of equations that are simply coherent.

\section{Hawkins (1989 a, b)}

He implements two non-associative basic processes: habituation and sensibilization. He also includes a type of associative sensitization.

The system generates responses that are proportional to the intensity of the stimuli it receives, and does not start from a state of behavioral silence. It shows Type $\alpha$ conditioning.

\section{Gluck \& Thompson (1987)}

They include only one form of non-associative learning: habituation. They also implement the associative sensitization form.

The only criterium that causes an answer is the presence or absence of a stimulus. The sensory pathways are initially unable to provoke a response, except for the nonconditioned fixed pathway. It shows Type

\section{B.conditioning}

The non-conditioned pathway is established functionally, so that any stimulus can be NS; it The non-conditioned pathway is "pre-wired". only depends on its intensity.

The interneuron does not directly stimulate the
motoneuron.

The interneuron enters an accomodation state, in

which it stops generating action potentials if the The refractory period in which the interneuron stimulation level does not exceed previous can enter does not turn it silent. values.

The model is not additive. The increases in the

It is an additive model: $P S P(t)=C_{4} \times \Sigma I_{\text {Caj }}(t) ; j \in$ s. basal

The eligibility period is determined by the previous levels of synaptic activation. somatic activation are identical as long as there is one or more excitatory postsynaptic potentials:

$$
\Delta A(t)=\delta_{1}(1-A(t)) \text { si } \exists j \mid P_{j}(t)=1
$$

The eligibility is determined by a function that depends only on the moment on which the stimulus presents itself, and which is independent from the activation values of the synapse.

Table 1. Some of the fundamental differences between the Gluck \& Thompson (1987) model and the Hawkins model (1989 a, b). 
The $\mathrm{H}_{\mathrm{m}}$, also based on the $\mathrm{HK}_{\mathrm{m}}$, satisfactorily covers the set of phenomena in Aplysia whose behavior is described, and many other phenomena which have not been verified yet (i.e., the model has a great heuristic value for the psychological and neurobiological research, since it describes phenomena expected in a system with specific properties). One of the most interesting characteristics of this model is its capacity to simulate a wide range of phenomena by using the same set of constants, whereas in the case of the $\mathrm{GT}_{\mathrm{m}}$ the constants must be adjusted if we want to obtain certain phenomena (e.g., we obtain an incipient form of blocking, only during the first compound Tests, and in such an unstable manner that it may not appear in ulterior simulations -its probabilistic character only maintains the 'strong' effects in the course of multiple sessions). This dichotomy between the approach of the $\mathrm{GT}_{\mathrm{m}}$ and the $\mathrm{H}_{\mathrm{m}}$ shows the importance of the integration level proposed by David Marr (1982).

These computational models have shown us the Aplysia's simple learning phenomena, and the architecture of the developed computational model has become a very interesting tool for the research of learning phenomena from a psychological, neurological or combined perspective. However, the role of the modelling techniques of an ANN should not be seen as a merely instrumental factor. Through its integration with the neurobiological and behavioral models of associative learning, the modelling encounters a series of problems that are usually beyond its habitual application field, which means that it not only opens up its horizon but also learns to redefine already known procedures and architectures:

- Although one of the most relevant behavioral models in the field, the Rescorla and Wagner (1972) model, expresses itself in the same way as an important rule in the ANN modelling field (i.e., the Widrow-Hoff rule, LMS), an implementation that is completely within this model does not require this rule. Rules that are effective at the behavioral level may not be so at the synaptic level (unless one single node is being used); in short, Rescorla and Wagner (1972), and Widrow-Hoff belong to different descriptive levels, and they must be treated as such.

- The type of ANN models that can adequately predict associative phenomena in living beings go beyond one of the classical dichotomies of the field, supervized learning versus non-supervised learning. The nonsupervized and self-organising models cannot implement them, and the supervized models, which are apparently coherent with Rescorla and Wagner (1972) lack the required behavioral richness. The models presented here are coherent with some of the principles of the Klopf-Kosko rule (Klopf, 1988): We 
introduce various concepts such as impulse, reinforcement, or the generation of internal signals that modulate the learning by taking up the role that is usually reserved for the external tutors.

- The most recommended application field for these architectures is the field of temporal, multivaried (i.e., each possible stimulus represents a variable that adopts different values in the course of time) and nonstationary series. The goal of these systems is to find correlational regularities (i.e., causal relationships) between the different variables-stimuli, so that a set of them can serve as temporally refined predictors of another set.

This work focuses on the considerable importance of computational models for the experimental research carried out in diverse science branches. It also proposes and develops an ideal computational model for experimentation in the field of psycho-neurobiological learning. This model was satisfactorily used during the verification of an ample set of theories and hypotheses related to diverse learning phenomena in the marine invertebrate Aplysia Californica. More specifically, we took Gluck's and Thompson's as well as Hawkins' learning models as the basis for new relationships, and we answered adequately a series of new questions thanks to the application of computational modelling.

\section{REFERENCES}

D. Bailey, J. Feldman, S. Narayanan and G. Lakoff, 1997, Modeling Embodied Lexical Development. Proceedings of the Nineteenth Annual Meeting of the Cognitive Science Conference, Stanford.

Baxter, D.A., Buonomano, D.V., Raymond, J.L., Cook, D.G., Kuenzi, F.M., Carew, T.J., and Byrne, J.H., 1991, Empirically derived adaptative elements and networks simulate associative learning. In M.L. Commons, S. Grossberg and J.E.R. Staddon (eds) Neural Network Models of Conditioning and Action. (Hillsdale, NJ: LEA).

Bunge, S.A., Mauelshagen, J., and Carew T.J., 1997, Reversal of relative thresholds for synaptic facilitation and increased excitability induced by serotonin and tail nerve stimulation in Aplysia sensory neurons. Neurobio. Learn. Memory, 67(3): 259-263.

Byrne, J.H., Gingrich, K.J., and Baxter, D.A., 1989, Computational capabilities of single neurons: Relationships to simple forms of associative and nonassociative learning in Aplysia. In R.D Hawkins and G.H. Bower (eds) Computational Models of Learning in Simple Systems. The Psychology of Learning and Motivation Vol 23 (San Diego CA: Academic Press), pp. 31-108. 
Emptage, N.J., Mauelshagen, J., and Carew, T.J., 1996, Threshold serotonin concentration required to produce synaptic facilitation differs for depressed and nondepressed synapses in Aplysia sensory neurons. J. Neurophysiol., 75: 843-854.

Gluck, M.A., and Thompson, R.F., 1987, Modeling the neural substrates of associative learning and memory: A computational approach. Psychological Review, 94:176-191.

Groves, P.M., and Thompson, R.F., 1970, Habituation: A dual-process theory. Psychological Review, 77: 419-450.

Hawkins, R.D., 1989a, A biologically realistic neural network model for higher-order features of classical conditioning. In R.G.M. Morris (ed.) Parallel Distributed Processing. Implications for Psychology and Neurobiology (New York: Oxford University Press), pp. 214-247

Hawkins, R.D., 1989b, A biologically based computational model for several forms of learning. In R.D. Hawkins and G.H. Bower (eds) Computational Models of Learning in Simple Systems. The Psychology of Learning and Motivation Vol 23 (San Diego CA: Academic Press), pp. 65-108.

Hawkins, R.D., Kandel E.R., 1984, Is there a cell biological alphabet for simple forms of learning? Psychological Review, 91: 375-391.

Hebb, D. O., 1949, The Organization of Behavior (NY: J. Wiley).

Klopf, A.H., 1988, A neuronal model for classical conditioning. Psychobiology, 16: 85-125.

Marr, D., 1982, Vision (San Francisco: W.H. Freeman).

Miller, R.R., and Matzel, L.D., 1989, Contingency and relative associative strength. In S.B. Klein and R.R. Mowrer (eds) Contemporary learning theories: Pavlovian conditioning and the status of traditional learning theory (Hillsdale, NJ: LEA), pp. 61-84

Pfautz, P.L., and Wagner, A.R., 1976, Transient variations in reponding to Pavlovian conditioned stimuli have implcations for mechanisms of 'priming'. Aminal Learning and Behavior, 4: 107-112.

Rescorla, R., 1968, Probability of shock in the presence and absence of CS in fear conditioning. Journal of Comparative and Physiological Psychology, 66: 1-5.

Rescorla, R.A., and Wagner, A.R., 1972, A theory of Pavlovian conditioning: Variations in the efectiveness of reinforcement and nonreinforcement. In A.H. Black and W.F. Prokasy (eds) Classical Conditioning II (New York: Appleton-Century-Crofts), pp. 64-99. 
Rumbaugh, J., 1991, Object-oriented modelling and design (New Jersey: Englewood Cliffs, Prentice-Hall)

Stopfer, M., Chen, X., Tai, U., Huang, G., and Carew, T.J., 1996, Site specificity of short-term and long-term habituation in the tail elicited siphon withdrawal reflex of Aplysia. J. Neurosci., 16: 4923-4932.

Wagner, A.R., 1976, Priming in STM: An information-processing mechanism for self-generated depression in performance. In T.J. Tighe and R.N. Leaton (eds) Habituation: Perspectives from child development, animal bahavior, and neurophysiology (Hillsdale, NJ: Erlbaum).

Wagner, A. R., 1979, Habituation and memory. In A. Dickinson and R.A. Boakes (eds) Mechanims of learning and motivation (Hillsdale, NJ: Erlbaum).

Wright, W.G., and Carew, T.J., 1995, A single identified interneuron gates tail-shock induced inhibition in the siphon withdrawal reflex of Aplysia. J. Neuosci., 15: 790-797. 